\title{
A CENSUS OF ROTATION AND VARIABILITY IN L1495: A UNIFORM ANALYSIS OF TRANS-ATLANTIC EXOPLANET SURVEY LIGHT CURVES FOR PRE-MAIN-SEQUENCE STARS IN TAURUS
}

\author{
Hong Yu Xiao ${ }^{1}$, Kevin R. Cover $^{1,6}$, Luisa Rebull ${ }^{2}$, David Charbonneau $^{3}$, Georgi Mandushev ${ }^{4}$, \\ Francis O’Donovan ${ }^{5}$, CATHerine Slesnick ${ }^{5}$, And James P. Lloyd ${ }^{1}$ \\ ${ }^{1}$ Department of Astronomy, Cornell University, 226 Space Sciences Building, Ithaca, NY 14853, USA \\ ${ }^{2}$ Spitzer Science Center/Caltech, M/S 220-6, 1200 East California Boulevard, Pasadena, CA 91125, USA \\ ${ }^{3}$ Department of Astronomy, Harvard University, 60 Garden Street, Cambridge, MA 02138, USA \\ ${ }^{4}$ Lowell Observatory, 1400 West Mars Hill Road, Flagstaff, AZ 86001, USA \\ ${ }^{5}$ Astronomy Department, California Institute of Technology, 1200 East California Boulevard, Pasadena, CA 91125, USA \\ Received 2012 January 6; accepted 2012 July 10; published 2012 August 28
}

\begin{abstract}
We analyze light curves obtained by the Trans-atlantic Exoplanet Survey (TrES) for a field centered on the L1495 dark cloud in Taurus. The Spitzer Taurus Legacy Survey catalog identifies 179 bona fide Taurus members within the TrES field; 48 of the known Taurus members are detected by TrES, as well as 26 candidate members identified by the Spitzer Legacy team. We quantify the variability of each star in our sample using the ratio of the standard deviation of the original light curve $\left(\sigma_{\text {orig. }}\right)$ to the standard deviation of a light curve that has been smoothed by 9 or 1001 epochs $\left(\sigma_{9}\right.$ and $\sigma_{1001}$, respectively). Known Taurus members typically demonstrate $\left(\sigma_{\text {orig. }} / \sigma_{9}\right)<2.0$, and $\left(\sigma_{\text {orig. }} / \sigma_{1001}\right)<5$, while field stars reveal $\left(\sigma_{\text {orig. }} / \sigma_{9}\right) \sim 3.0$ and $\left(\sigma_{\text {orig. }} / \sigma_{1001}\right) \sim 10$, as expected for light curves dominated by unstructured white noise. Of the 74 Taurus members/candidates with TrES light curves, we detect significant variability in 49 sources. Adapting a quantitative metric originally developed to assess the reliability of transit detections, we measure the amount of red and white noise in each light curve and identify 18 known or candidate Taurus members with highly significant period measurements. These appear to be the first periods measured for four of these sources (HD 282276, CX Tau, FP Tau, TrES J042423+265008), and in two other cases, the first non-aliased periods ( $\mathrm{LkCa} 21$ and DK Tau AB). For the remainder, the TrES measurements typically agree very well $(\delta P<1 \%)$ with previously reported values. Including periods measured at lower confidence for 15 additional sources, we report periods for 11 objects where no previous periods were found, including 8 confirmed Taurus members. We also identify 10 of the 26 candidate Taurus members that demonstrate variability levels consistent with being bona fide T Tauri stars. A Kolomgorov-Smirnov (K-S) test confirms that these new periods confirm the distinction between the rotation period distributions of stars with and without circumstellar disks, with only a $10 \%$ probability of the two populations sharing the same parent period distribution. K-S tests do suggest, however, that the updated Taurus period distribution now more closely resembles those measured in other young star-forming clusters (i.e., NGC 2264, NGC 6530, and the ONC). This improved agreement may reflect the exclusion of long rotation periods which are detected in Taurus at lower significance, and which may be beyond the limits of detectability in more distant star-forming regions.
\end{abstract}

Key words: stars: pre-main sequence - stars: rotation

Online-only material: color figures, figure set

\section{INTRODUCTION}

As Scholz (2012) documented, T Tauri stars have been observed to be variable for more than a century (Knott 1891), and optical variability was identified as one of the T Tauri stars' defining characteristics (Joy 1945). Multi-epoch photometry of young stellar objects (YSOs) therefore provides the opportunity to measure the characteristic timescales and amplitudes of short-timescale processes associated with the formation of stars and planets, such as changes in a star's mass accretion rate (Hartmann et al. 1993; Miller et al. 2011) and transient obscuration by circumstellar dust (Hamilton et al. 2001; Covey et al. 2011; Hillenbrand et al. 2012).

The photometric variability induced by star spots on the stellar photosphere is perhaps the most ubiquitous type of variability exhibited by optically revealed pre-main-sequence stars. Analyses of these photometric variations have both revealed the properties of the star spots themselves (e.g., Vrba et al. 1988), and

\footnotetext{
6 Hubble Fellow.
}

enabled the measurement of the star's (surface) rotation period from the characteristic timescale of these variations (Rydgren \& Vrba 1983). Studies of stellar rotation periods measured in young clusters then provide a basic portrait of the evolution of a star's angular momentum content during the pre-main-sequence phase. These analyses suggest that several processes may modify a young star's angular momentum content, including magnetospheric accretion, outflow activity, and magnetic and dynamical interactions with circumstellar disks and stellar or planetary companions. The sum total of these processes seems to drive young stars to corotate with the inner edge of their circumstellar disk while the disk is present, and then to spin up as the star's radius (and thus moment of inertia) continues to shrink following the dissipation of the disk. This qualitative understanding of pre-main-sequence angular momentum evolution is far from complete, however; we still lack a clear understanding of the relative roles these physical mechanisms play in determining a young solar-type star's equilibrium rotation rate (if the star reaches equilibrium at all), much less how their importance changes as a function of stellar mass or evolutionary stage. 
Improving our understanding of the angular momentum content and evolution of pre-main-sequence stars therefore requires that we assemble large samples of empirically measured rotation periods for pre-main-sequence stars spanning a range of masses and evolutionary stages. To this end, we have analyzed the variability of $\mathrm{T}$ Tauri stars using archival data from two large-scale surveys: the Trans-atlantic Exoplanet Survey (TrES; Alonso et al. 2007) and the Taurus Spitzer Survey (TSS; Rebull et al. 2010). Both surveys provide coverage of the L1495 dark cloud (Lynds 1962), which contains the most populous sub-cluster within the Taurus star-forming region (Kenyon et al. 2008). The TrES database has been extensively analyzed to identify transiting exoplanets (e.g., Alonso et al. 2004a; O'Donovan et al. 2006, 2007; Mandushev et al. 2007) and eclipsing binaries (e.g., Devor et al. 2008a, 2008b), but the densely sampled TrES light curves also contain valuable information about periodic and aperiodic stellar variability. This promise is demonstrated by several recent studies of stellar rotation based upon analyses of light curves obtained by various exoplanet transit searches (e.g., Hartman et al. 2008; Collier Cameron et al. 2009; Irwin et al. 2011; Delorme et al. 2011). L1495 is an equally promising target for such an analysis, given the large spot sizes and photometric amplitudes that characterize typical $\mathrm{T}$ Tauri stars and the importance of assembling a large database of pre-main-sequence rotation measurements to inform our understanding of stellar angular momentum evolution at the youngest ages.

We have analyzed the variability of young stars in L1495 as captured by TrES monitoring data obtained from 2005 December through 2006 January. In Section 2, we briefly summarize the TrES and TSS photometric catalogs, and describe how we have used the Spitzer catalog to identify 74 known or candidate Taurus members with TrES light curves. We present our analysis of the TrES light curves in Section 3, whose primary aim is to measure stellar rotation periods from repeated photometric variations. We describe our results in Section 4 and summarize our conclusions in Section 5.

\section{PHOTOMETRIC CATALOGS}

The data we analyze here have been presented previously in studies addressing different science goals. Rather than exhaustively describing each data set, we briefly summarize their most relevant characteristics while directing the interested reader to more extensive discussions of each data set in the literature.

\subsection{TrES Observations of L1495}

The TrES survey was carried out by a network of three $10 \mathrm{~cm}$ telescopes: the Sleuth telescope at Mount Palomar, CA (O'Donovan et al. 2004), the Planet Search Survey Telescope (PSST; Dunham et al. 2004) at Lowell Observatory, AZ, and the STellar Astrophysics and Research on Exoplanets telescope (STARE; Alonso et al. 2004b), on the island of Tenerife, in the Canary Islands, Spain. Each TrES telescope was equipped with a $2 \mathrm{~K} \times 2 \mathrm{~K} \mathrm{CCD}$ with $13 \mu \mathrm{m}$ pixels, producing a plate scale of $\sim 10^{\prime \prime}$ pixel $^{-1}$ and photometric apertures of $\sim 30^{\prime \prime}$. With fields of view of roughly $5.75 \times 5.75$, each TrES telescope detected $10-25$ thousand bright $(9.5 \leqslant V \leqslant 15.5)$ stars in a typical field. The TrES telescopes operated in a semi-automated fashion to monitor a common set of fields until $\geqslant 80 \%$ sensitivity to planetary transits with periods $\sim 5$ days had been achieved, which typically required observing campaigns lasting one to three months. The TrES Taurus campaign monitored a field containing L1495 for 69 days, from UT 2005 December 1 through UT 2006 February 3. Observations were taken on an approximately two-minute cadence, weather and observing conditions permitting. Light curves were subsequently rebinned to a resolution of 9 minutes per data point, with light curves for most target stars containing $\sim 10,000$ data points.

The Taurus data analyzed here were primarily obtained by Sleuth and PSST. Data from each TrES telescope were reduced independently, following the reduction procedures described in full by Dunham et al. (2004), Mandushev et al. (2005), and O'Donovan et al. (2006, 2007). In brief, stars were detected in each individual frame using a modified version of the DAOPHOT II package (Stetson 1987, 1992), with aperture photometry measured for each star from a difference image produced by subtracting each target image from a master reference frame with the ISIS code (Alard 2000). Differential magnitudes $(\Delta m)$ were calculated for the $i$ th epoch of each star as

$$
\Delta m_{i}=-2.5 \log \left[\left(F_{0}-\Delta F_{i}\right) / F_{0}\right],
$$

where $F_{0}$ is the star's flux in the reference image and $\Delta F_{i}$ is the residual flux measured in the aperture on the $i$ th difference image. ${ }^{7}$ Intrinsically low root-mean-squared (rms) light curves were detrended by subtracting a least squares fit to the photometry of all non-variable stars of similar brightness in a given field from each target star whose light curve possessed an rms below a certain magnitude-dependent threshold (which excluded high-amplitude variable stars). Light curves from each TrES telescope were then merged to create a unified database of TrES photometry for a given field: the merged TrES database includes 15,779 objects in the Taurus field, of which we analyze the brightest 14,000 here.

\subsection{Taurus Spitzer Survey}

The TSS utilized the Infrared Array Camera (IRAC; Fazio et al. 2004) and Multi-band Imaging Photometer for Spitzer (MIPS; Rieke et al. 2004) on the Spitzer Space Telescope (Werner et al. 2004) to image $44 \mathrm{deg}^{2}$ of the Taurus molecular cloud at wavelengths spanning 3.6-160 $\mu \mathrm{m}$ (Padgett et al. 2008; D. Padgett et al. 2012, in preparation). Rebull et al. (2010) used these observations to select candidate Taurus members, and to generate a catalog of Spitzer confirmed and candidate Taurus members within the Legacy Survey footprint, which we adopt in our analysis.

Rebull et al. (2010) provide a full description of the construction of their Taurus catalog: we briefly summarize here the aspects most pertinent to our analysis. IRAC and MIPS images of Taurus were processed using the MOsaicking and Point source EXtractor (MOPEX; Makovoz \& Marleau 2005). Photometry was performed on the IRAC and MIPS mosaics using aperture and point-response function fitting techniques, respectively, and adopting standard zero points given by the relevant instrument handbooks and Spitzer Science Center Web site. Spitzer and Two Micron All Sky Survey (2MASS; Skrutskie et al. 2006) photometry were bandmerged by first matching sources detected at wavelengths between 2 and $8 \mu \mathrm{m}$ with a $1^{\prime \prime}$ matching radius. Detections at 24 and $70 \mu \mathrm{m}$ were then merged using a $10^{\prime \prime}$ matching radius, and then merged to the shorter wavelength catalog by applying a $2^{\prime \prime}$ matching radius between the source positions at $24 \mu \mathrm{m}$ and shorter wavelengths.

\footnotetext{
Differential magnitudes produced by this procedure behave as expected: a star which is brighter in the current epoch than in the template image produces a negative $\Delta m$, and vice versa.
} 
This merged 2MASS/Spitzer catalog was then supplemented with observations at shorter wavelengths, incorporating optical data from the Canada-France-Hawaii Telescope (Guieu et al. 2006; Güdel et al. 2007; Monin et al. 2010), Sloan Digital Sky Survey (Finkbeiner et al. 2004; Padmanabhan et al. 2008), and $\mathrm{UV} / \mathrm{X}$-ray data from the XMM-Newton Extended Survey of the TMC program (e.g., Audard et al. 2007; Güdel et al. 2007).

Rebull et al. (2010) identified 215 known Taurus members within the footprint of the TSS by merging previous membership catalogs compiled by Güdel et al. (2007) and Kenyon et al. (2008), and verified that Spitzer observations detected all previously identified Taurus members within the TSS footprint, even those sources that lack mid-IR excesses indicative of the presence of circumstellar disks. Rebull et al. (2010) then analyzed their merged catalog of Spitzer detections to identify 148 additional sources with photometric properties consistent with a YSO classification. Spectroscopic follow-up of roughly half of the sample confirmed 34 of these objects as bona fide $\mathrm{T}$ Tauri stars, with 106 requiring additional follow-up data to confirm or reject their status as candidate cluster members. In total, Rebull et al. (2010) list 249 confirmed Taurus members within the Spitzer Legacy footprint, with 179 lying within the footprint of the TrES L1495 field.

\subsection{Bulk Photometric Properties of Candidate and Confirmed Taurus Members with Spitzer/TrES Detections}

We identified TrES light curves for candidate and confirmed Taurus members by crossmatching the TSS catalog with that of the TrES L1495 field. Using a matching radius of $5^{\prime \prime}$, we identified 8049 sources in the L1495 field detected by both Spitzer and TrES. Restricting this TrES/Spitzer catalog to Taurus members tabulated by Kenyon et al. (2008) and Rebull et al. (2010) generated a sample of 74 confirmed or candidate Taurus members with TrES/Spitzer detections. Rebull et al. (2010) classify 48 of these stars as previously known or newly confirmed members; of the remaining 26, 4 and 22 are classified as "possible members" and "pending investigation," respectively. All 74 confirmed and candidate Taurus members detected by TrES are listed in Table 1 and shown in Figure 1. For context, Figure 1 also displays the full catalog of TrES detections in the L1495 field, and highlights the locations of Taurus candidates that were not recovered by TrES.

To verify the membership and evolutionary status of the confirmed and candidate Taurus members detected in the TrES Taurus field, we display in Figures 2 and 3 the distribution of TrES/Spitzer detections in $r-K_{s}$ versus $K_{s}$ and [3.6]-[8] versus $r$ color-magnitude spaces, respectively. The known or candidate Taurus members identified in Figure 2 are significantly redder and brighter than the field star population, as expected given the proximity and elevated luminosities and reddenings characteristic of pre-main-sequence stars in the nearby Taurus molecular cloud. The [3.6]-[8] versus $r$ color-magnitude diagram in Figure 3 demonstrates the relatively wide separation between YSOs with circumstellar disks (Class II YSOs in the classification scheme of Lada 1987, identified via IRAC colors as [3.6]-[8] > 1.0; Rebull et al. 2006), and YSOs without circumstellar disks ([3.6]-[8] $<1.0$; Class III YSOs in the scheme of Lada 1987). The distribution of TrES/Spitzer-detected known and candidate Taurus members in this color-magnitude space indicates that $\sim 1 / 3$ of the YSOs detected by TrES possess NIR excesses indicative of circumstellar disks, while the remaining $\sim 2 / 3$ exhibit no evidence of an optically thick dust disk.

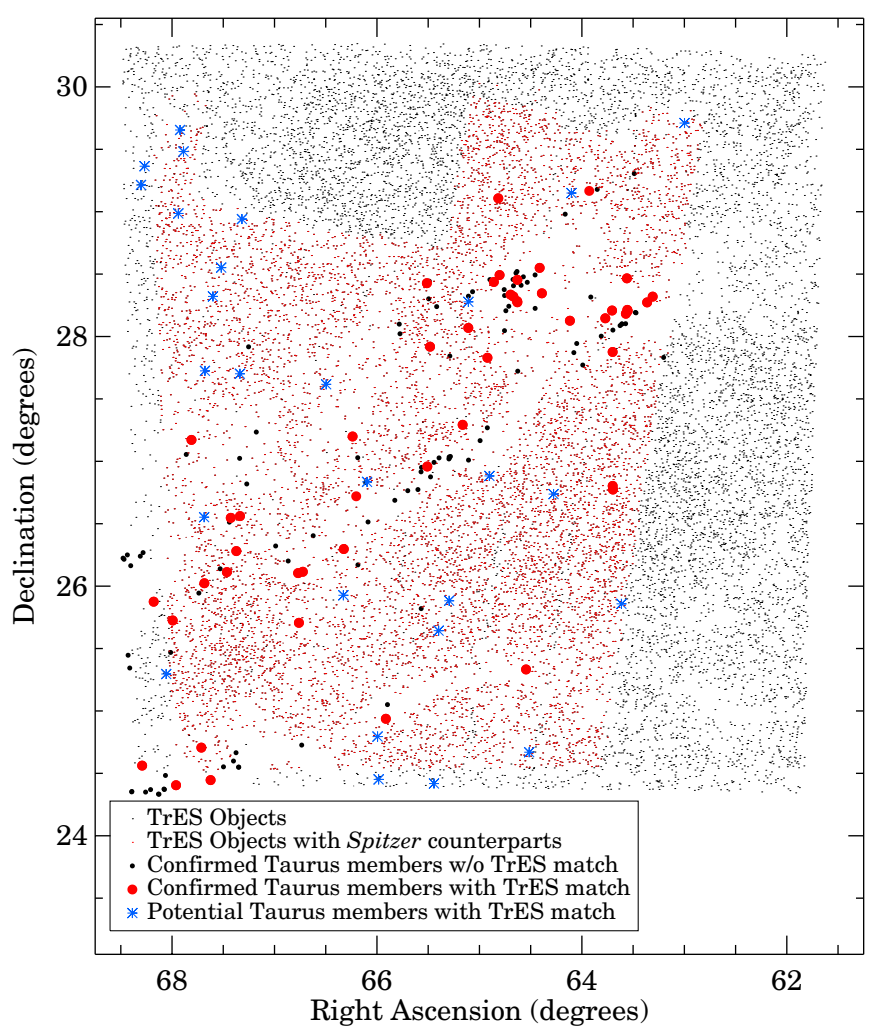

Figure 1. TrES and Spitzer detections in the L1495 TrES field. The coverage of the Spitzer Legacy Survey is visible as the regions where TrES objects typically possess Spitzer counterparts, while filamentary structures in the Taurus molecular cloud are also visible as areas with few TrES detections. Taurus members without TrES counterparts are predominantly embedded within these filaments, while most off-filament Taurus sources are detected by TrES.

(A color version of this figure is available in the online journal.)

\section{LIGHT-CURVE ANALYSIS}

\subsection{Identifying Variable Stars}

We adopt a simple criterion to discriminate between optically variable and non-variable sources: the standard deviations of the original and smoothed TrES light curves.

The light curves of non-variable stars are dominated by unstructured photometric noise, at least in the absence of correlated errors (i.e., red noise). Smoothing such light curves by $n$ epochs should therefore reduce their standard deviations by $1 / \sqrt{n}$. Variable stars, however, have light curves that are dominated by temporal structure; smoothing a variable star's light curve will not reduce the light curve's standard deviation by a factor of $1 / \sqrt{n}$, as long the underlying structure has a typical timescale that exceeds the temporal width of the smoothing window. The ratio of the standard deviations of a smoothed and unsmoothed light curve can therefore distinguish between stars whose light curves do and do not contain temporally structured variations. TrES light curves do contain some level of red noise, such that we expect these light curves will not compress exactly as $1 / \sqrt{n}$, but the variability exhibited by a standard T Tauri star is sufficiently large that intrinsic temporal variations will typically dominate the red-noise effects.

We have therefore calculated the standard deviation for each TrES light curve, both in its original form, and when boxcar smoothed by 9 and 1001 (consecutive) epochs. In the uniformly sampled limit, smoothing by 9 and 1001 epochs corresponds to temporal filters tuned to variability on timescales of hours 
Table 1

Period Measurements for TrES-detected Taurus Members

\begin{tabular}{|c|c|c|c|c|c|c|c|c|c|c|c|c|c|c|}
\hline Name & $\begin{array}{l}\text { J2000 } \\
\text { R.A. } \\
\text { (deg) }\end{array}$ & $\begin{array}{c}\text { J2000 } \\
\text { Decl. } \\
\text { (deg) }\end{array}$ & $\begin{array}{c}r \\
\text { Mag } \\
(\mathrm{mag})\end{array}$ & $\begin{array}{l}\text { White } \\
\text { Noise } \\
\text { (mag) }\end{array}$ & $\begin{array}{c}\text { Red } \\
\text { Noise } \\
\text { (mag) }\end{array}$ & $\begin{array}{c}\text { Lit. } \\
\text { Period }^{\text {a }} \\
\text { (days) }^{\text {days }}\end{array}$ & $\begin{array}{c}\text { Lit. } \\
\operatorname{Ref}^{\mathrm{a}, \mathrm{b}}\end{array}$ & $\begin{array}{l}\text { TrES } \\
\text { Period } \\
\text { (days) }\end{array}$ & $\begin{array}{c}\text { Per. } \\
\text { Signif. } \\
\left(S_{T}\right)\end{array}$ & $\begin{array}{c}\text { Per. } \\
\text { Ampl. } \\
\text { (mag) }\end{array}$ & $\begin{array}{l}\text { Peak } \\
\text { Power }\end{array}$ & $\begin{array}{c}\text { Power } \\
\sigma\end{array}$ & $\begin{array}{l}\text { Spec. } \\
\text { Type }\end{array}$ & $\begin{array}{c}\text { Member } \\
\text { Status }\end{array}$ \\
\hline \multicolumn{15}{|c|}{ "Definitely Periodic" Stars } \\
\hline HD 282276 & 68.267917 & 29.364167 & 11.66 & 0.005 & 0.004 & & & 3.115 & 17.5 & 0.012 & 531 & 13.5 & B8 & $\mathrm{C}$ \\
\hline V773 Tau ABC & 63.553750 & 28.203333 & 11.76 & 0.042 & 0.036 & $(3.43) / 3.08 / 51.5$ & (BJ)FK & 3.075 & 13.5 & 0.191 & 3598 & 14.99 & K2 & M \\
\hline V410 Tau ABC & 64.629583 & 28.454167 & 12.12 & 0.065 & 0.060 & $(1.87) / 1.92$ & (ACEI)J & 1.869 & 11.9 & 0.225 & 3453 & 13.30 & K4 & $\mathrm{M}$ \\
\hline LkCa 7 AB & 64.921667 & 27.829722 & 13.31 & 0.018 & 0.015 & $(5.65) / 6.0$ & (ACFG)E & 5.667 & 58.2 & 0.283 & 4912 & 12.11 & K7 & $\mathrm{M}$ \\
\hline V830 Tau & 68.291667 & 24.561944 & 13.32 & 0.023 & 0.021 & $(2.75) / 2.8$ & $(\mathrm{ACGJ}) \mathrm{E}$ & 2.747 & 85.5 & 0.160 & 2035 & 11.62 & M1 & M \\
\hline Hubble 4 & 64.695833 & 28.335278 & 13.43 & 0.015 & 0.012 & $3.37 / 1.5483$ & $\mathrm{BF}$ & 1.547 & 9.2 & 0.04 & 2670 & 16.79 & K7 & M \\
\hline DK Tau AB & 67.684583 & 26.023333 & 13.56 & 0.166 & 0.160 & $8.40 / 8.18$ & $\mathrm{CE}$ & 4.094 & 12.3 & 0.72 & 2098 & 8.01 & M0 & M \\
\hline $\mathrm{LkCa} 4$ & 64.117083 & 28.126389 & 13.60 & 0.067 & 0.062 & $(3.37) /-99.0$ & $(\mathrm{ACH}) \mathrm{E}$ & 3.371 & 24.7 & 0.58 & 4760 & 15.12 & K7 & M \\
\hline DG Tau A & 66.769583 & 26.104444 & 13.62 & 0.063 & 0.061 & $6.30 /-99.0 /-99.0$ & CBE & 3.652 & 8.1 & 0.177 & 2140 & 8.92 & K5 & M \\
\hline V819 Tau & 64.859167 & 28.436944 & 13.92 & 0.017 & 0.013 & $(5.55)$ & (ACEGJ) & 5.507 & 37.7 & 0.165 & 4733 & 11.76 & K7 & M \\
\hline Anon $1^{\mathrm{c}}$ & 63.363333 & 28.273333 & 14.19 & 0.020 & 0.015 & $6.493 / 0.8637$ & $\mathrm{AF}$ & 6.454 & 7.4 & 0.048 & 1393 & 10.02 & M0 & M \\
\hline $\mathrm{LkCa} 21$ & 65.512917 & 28.427222 & 14.39 & 0.026 & 0.021 & $8.80 / 8.6$ & $\mathrm{CE}$ & 0.915 & 12.1 & 0.054 & 2051 & 14.00 & M3 & M \\
\hline $\operatorname{LkCa} 5^{\mathrm{c}}$ & 64.412083 & 28.550000 & 14.57 & 0.021 & 0.015 & 1.4127 & $\mathrm{~F}$ & 1.411 & 6.5 & 0.035 & 1380 & 18.80 & M2 & M \\
\hline CX Tau & 63.699583 & 26.803056 & 14.69 & 0.024 & 0.018 & $\ldots$ & $\ldots$ & 3.302 & 8.0 & 0.053 & 2082 & 14.59 & M1.5 & M \\
\hline FP Tau & 63.697083 & 26.773889 & 14.76 & 0.033 & 0.026 & $\ldots$ & $\ldots$ & 2.194 & 10.2 & 0.083 & 2146 & 14.27 & M2.5 & M \\
\hline $\mathrm{LkCa} 1^{\mathrm{c}}$ & 63.309167 & 28.319444 & 14.77 & 0.028 & 0.020 & $(2.5)$ & (AG) & 2.494 & 2.8 & 0.036 & 384 & 15.67 & M4 & M \\
\hline DD Tau AB & 64.629583 & 28.274444 & 15.78 & 0.158 & 0.152 & $5.50 / 5.7593$ & $\mathrm{EF}$ & 5.706 & 10.6 & 0.52 & 2689 & 9.57 & K6 & M \\
\hline TrES J042423+265008 & 66.096667 & 26.835556 & 16.40 & 0.084 & 0.062 & $\ldots$ & $\ldots$ & 7.094 & 9.1 & 0.25 & 2796 & 11.09 & M3 & $\mathrm{C}$ \\
\hline \multicolumn{15}{|c|}{ "Variable, Possibly Periodic" Stars } \\
\hline HD 283663 & 65.983750 & 24.451389 & 12.54 & 0.008 & 0.006 & . & $\ldots$ & 0.392 & 5.3 & 0.01 & 210 & 12.97 & $\mathrm{~F} 2$ & $\mathrm{C}$ \\
\hline $\mathrm{LkCa} 3 \mathrm{AB}^{\mathrm{d}}$ & 63.700000 & 27.876111 & 13.03 & 0.014 & 0.011 & $(7.36) / 7.2$ & $(\mathrm{AF}) \mathrm{B}$ & 3.689 & 5.3 & 0.022 & 1081 & 9.42 & M1 & M \\
\hline ВР Тau & 64.815833 & 29.107222 & 13.41 & 0.068 & 0.064 & $7.60 / 7.7 / 24.0$ & $\mathrm{CDE}$ & 8.309 & 7.9 & 0.302 & 2576 & 7.39 & K7 & $\mathrm{M}$ \\
\hline DI Tau $\mathrm{AB}^{\mathrm{d}}$ & 67.426667 & 26.547222 & 13.56 & 0.039 & 0.037 & $7.50 /-99.0 / 7.64$ & CEG & 7.733 & 6.3 & 0.097 & 1283 & 3.68 & M0 & M \\
\hline CW Tau & 63.570833 & 28.182500 & 13.75 & 0.292 & 0.285 & 8.25 & $\mathrm{~B}$ & 5.958 & 7.9 & 0.928 & 1660 & 6.82 & K5 & M \\
\hline DE Tau & 65.481667 & 27.918333 & 13.88 & 0.027 & 0.023 & 7.60 & $\mathrm{C}$ & 18.221 & 4.3 & 0.061 & 960 & 5.68 & M1 & M \\
\hline V409 Tau & 64.545000 & 25.332500 & 14.21 & 0.154 & 0.150 & $\ldots$ & $\ldots$ & 4.754 & 5.7 & 0.307 & 1383 & 4.33 & K8 & M \\
\hline IP Tau ${ }^{\mathrm{d}}$ & 66.237917 & 27.198889 & 14.22 & 0.103 & 0.099 & 3.25 & $\mathrm{C}$ & 5.586 & 9.9 & 0.322 & 1752 & 7.63 & M0 & M \\
\hline $\mathrm{IQTau}^{\mathrm{d}}$ & 67.464583 & 26.112500 & 14.357 & 0.123 & 0.118 & $6.25 / 12.5$ & $\mathrm{BD}$ & 6.902 & 9.2 & 0.446 & 1750 & 4.99 & M2 & M \\
\hline FX Tau AB & 67.623750 & 24.446111 & 14.47 & 0.072 & 0.070 & .. & $\ldots$ & 6.402 & 7.5 & 0.288 & 1557 & 5.07 & M4 & M \\
\hline $\mathrm{J} 1-4872 \mathrm{AB}$ & 66.323750 & 26.297222 & 14.52 & 0.027 & 0.022 & $\ldots$ & $\ldots$ & 6.942 & 6.5 & 0.060 & 1188 & 6.63 & K7 & M \\
\hline FM Tau & 63.556667 & 28.213333 & 15.24 & 0.340 & 0.288 & $\ldots$ & $\ldots$ & 3.073 & 6.2 & 1.22 & 1202 & 9.48 & $\mathrm{~K} 3$ & M \\
\hline TrES J042026+280407 & 65.108750 & 28.068611 & 15.61 & 0.038 & 0.027 & $\ldots$ & $\ldots$ & 2.854 & 7.5 & 0.057 & 1066 & 15.96 & M2 & M \\
\hline FT Tau $^{\mathrm{d}}$ & 65.913333 & 24.937222 & 15.77 & 0.138 & 0.132 & 8.30 & $\mathrm{D}$ & 5.238 & 11.2 & 0.51 & 1333 & 5.25 & M3 & M \\
\hline CZ Tau AB & 64.631667 & 28.282778 & 16.22 & 0.111 & 0.088 & $\ldots$ & $\ldots$ & 5.631 & 5.9 & 0.17 & 1445 & 9.98 & M2 & M \\
\hline
\end{tabular}




\begin{tabular}{|c|c|c|c|c|c|c|c|c|c|c|c|c|c|c|}
\hline$\overline{\text { Name }}$ & $\begin{array}{l}\text { J2000 } \\
\text { R.A. } \\
\text { (deg) }\end{array}$ & $\begin{array}{l}\text { J2000 } \\
\text { Decl. } \\
\text { (deg) }\end{array}$ & $\begin{array}{c}r \\
\text { Mag } \\
(\mathrm{mag})\end{array}$ & $\begin{array}{l}\text { White } \\
\text { Noise } \\
\text { (mag) }\end{array}$ & $\begin{array}{c}\text { Red } \\
\text { Noise } \\
\text { (mag) }\end{array}$ & $\begin{array}{c}\text { Lit. } \\
\text { Period }^{\text {a }} \\
\text { (days) }^{\text {days }}\end{array}$ & $\begin{array}{c}\text { Lit. } \\
\text { Ref }^{\mathrm{a}, \mathrm{b}}\end{array}$ & $\begin{array}{c}\text { TrES } \\
\text { Period } \\
\text { (days) }\end{array}$ & $\begin{array}{c}\text { Per. } \\
\text { Signif. } \\
\left(S_{T}\right)\end{array}$ & $\begin{array}{c}\text { Per. } \\
\text { Ampl. } \\
\text { (mag) }\end{array}$ & $\begin{array}{l}\text { Peak } \\
\text { Power }\end{array}$ & $\begin{array}{c}\text { Power } \\
\sigma\end{array}$ & $\begin{array}{l}\text { Spec. } \\
\text { Type }\end{array}$ & $\begin{array}{c}\text { Member } \\
\text { Status }\end{array}$ \\
\hline \multicolumn{15}{|c|}{ "Variable, Not Periodic" Stars } \\
\hline DF Tau AB & 66.761667 & 25.706111 & 12.80 & 0.194 & 0.189 & $8.5 / 9.8 /-99.0$ & $\mathrm{CBE}$ & $\ldots$ & 5.9 & 0.83 & 2400 & 5.82 & K5 & M \\
\hline GSC $01833-00754^{d}$ & 68.057083 & 25.295833 & 13.19 & 0.193 & 0.177 & $\ldots$ & $\ldots$ & $\ldots$ & 11.2 & 0.60 & 674 & 4.41 & A9 & $\mathrm{C}$ \\
\hline UZ Tau Aab ${ }^{\mathrm{e}}$ & 68.178750 & 25.875278 & 13.33 & 0.103 & 0.098 & $\ldots$ & $\ldots$ & $\ldots$ & 19.5 & 0.28 & 1931 & 4.29 & M1 & M \\
\hline $\mathrm{JH} 56^{\mathrm{e}}$ & 67.810417 & 27.171667 & 13.57 & 0.027 & 0.024 & 5.9087 & $\mathrm{~F}$ & $\ldots$ & 11.7 & 0.19 & 848 & 9.15 & M1 & M \\
\hline CY Tau & 64.390417 & 28.346111 & 14.40 & 0.057 & 0.054 & 7.50 & $\mathrm{~B}$ & $\ldots$ & 5.7 & 0.11 & 904 & 6.03 & M2 & M \\
\hline V412 Tau ${ }^{\mathrm{e}}$ & 65.295417 & 25.882778 & 14.64 & 0.108 & 0.098 & $\ldots$ & $\ldots$ & $\ldots$ & 12.2 & 0.63 & 2053 & 4.85 & M6 & $\mathrm{C}$ \\
\hline TrES J043043+274330 & 67.678750 & 27.725000 & 15.10 & 0.143 & 0.136 & $\ldots$ & $\ldots$ & $\ldots$ & 37.5 & 0.74 & 2280 & 4.50 & M6 & $\mathrm{C}$ \\
\hline TrES J042519+255536 & 66.327917 & 25.926667 & 15.27 & 0.050 & 0.042 & $\ldots$ & $\ldots$ & $\ldots$ & 16.1 & 0.17 & 2445 & 4.55 & M5 & $\mathrm{C}$ \\
\hline TrES J042146+242507 & 65.443333 & 24.418611 & 15.37 & 0.069 & 0.061 & $\ldots$ & $\ldots$ & $\ldots$ & 27.7 & 0.25 & 2145 & 4.18 & M3 & $\mathrm{C}$ \\
\hline $\mathrm{J} 1-507$ & 67.336667 & 26.561111 & 15.45 & 0.033 & 0.025 & $\ldots$ & $\ldots$ & $\ldots$ & 7.1 & 0.041 & 505 & 7.80 & M4 & M \\
\hline HK Tau $\mathrm{AB}^{\mathrm{f}}$ & 67.960833 & 24.405000 & 15.66 & 0.100 & 0.089 & $\ldots$ & $\ldots$ & $\ldots$ & 16.7 & 0.177 & 670 & 5.81 & M0.5 & M \\
\hline FO Tau AB & 63.705417 & 28.208333 & 15.85 & 0.055 & 0.042 & $\ldots$ & $\ldots$ & $\ldots$ & 4.2 & 0.106 & 602 & 4.61 & M2 & M \\
\hline IRAS $04262+2735^{\mathrm{e}}$ & 67.337500 & 27.701667 & 15.85 & 0.060 & 0.048 & $\ldots$ & $\ldots$ & $\ldots$ & 12.7 & 0.15 & 2992 & 4.56 & M5 & $\mathrm{C}$ \\
\hline TrES J043024+281916 & 67.600833 & 28.321111 & 16.03 & 0.098 & 0.079 & $\ldots$ & $\ldots$ & $\ldots$ & 10.9 & 0.22 & 3249 & 4.25 & M5 & $\mathrm{C}$ \\
\hline FV Tau AB & 66.723750 & 26.115278 & 16.04 & 0.072 & 0.058 & $\ldots$ & $\ldots$ & $\ldots$ & 7.2 & 0.16 & 517 & 5.68 & K5 & M \\
\hline FQ Tau AB & 64.803333 & 28.492222 & 16.06 & 0.166 & 0.150 & $\ldots$ & $\ldots$ & $\ldots$ & 4.17 & 0.42 & 750 & 6.88 & M2 & M \\
\hline \multicolumn{15}{|c|}{ "Not Variable" Stars } \\
\hline RXJ0424.8 & 66.202500 & 26.720000 & 11.73 & 0.010 & 0.0076 & $3.18 / 1.89$ & FK & $\ldots$ & 3.4 & 0.008 & 674 & 10.28 & K1 & M \\
\hline HD 282277 & 68.302917 & 29.214167 & 12.36 & 0.005 & 0.004 & $\ldots$ & $\ldots$ & $\ldots$ & 2.3 & 0.002 & 24 & 13.39 & A7 & $\mathrm{C}$ \\
\hline HD 281820 & 64.102083 & 29.149722 & 12.48 & 0.007 & 0.005 & $\ldots$ & $\ldots$ & $\ldots$ & 2.4 & 0.002 & 21 & 8.49 & A2 & $\mathrm{C}$ \\
\hline HD 283637 & 66.495417 & 27.616944 & 12.69 & 0.006 & 0.005 & $\ldots$ & $\ldots$ & $\ldots$ & 1.5 & 0.002 & 19 & 8.01 & A0 & $\mathrm{C}$ \\
\hline HD 283629 & 67.317917 & 28.941111 & 12.90 & 0.008 & 0.006 & $\ldots$ & $\ldots$ & $\ldots$ & 3.4 & 0.008 & 370 & 8.49 & G5 & $\mathrm{C}$ \\
\hline TrES J043045+263309 & 67.686667 & 26.552500 & 13.08 & 0.007 & 0.005 & $\ldots$ & $\ldots$ & $\ldots$ & 1.3 & 0.002 & 22 & 11.58 & K3 & $\mathrm{C}$ \\
\hline 2MASS J04180338+2440096 & 64.514167 & 24.669444 & 13.30 & 0.015 & 0.011 & $\ldots$ & $\ldots$ & $\ldots$ & 3.1 & 0.004 & 53 & 17.57 & A9 & $\mathrm{C}$ \\
\hline TrES J043133+292856 & 67.887917 & 29.482222 & 13.61 & 0.015 & 0.012 & $\ldots$ & $\ldots$ & $\ldots$ & 1.0 & 0.003 & 36 & 8.65 & B9 & $\mathrm{C}$ \\
\hline TrES J042026+281641 & 65.107917 & 28.278056 & 13.95 & 0.014 & 0.010 & $\ldots$ & $\ldots$ & $\ldots$ & 2.7 & 0.005 & 48 & 8.63 & G2 & $\mathrm{C}$ \\
\hline TrES J043145+285909 & 67.937917 & 28.985833 & 14.69 & 0.021 & 0.015 & $\cdots$ & $\ldots$ & $\cdots$ & 2.5 & 0.008 & 34 & 12.12 & F0 & $\mathrm{C}$ \\
\hline TrES J043005+283307 & 67.519583 & 28.551944 & 14.70 & 0.019 & 0.014 & $\ldots$ & $\ldots$ & $\ldots$ & 1.4 & 0.007 & 24 & 9.53 & $\mathrm{~K} 2$ & $\mathrm{C}$ \\
\hline TrES J042359+244742 & 65.994167 & 24.795000 & 15.05 & 0.026 & 0.019 & $\ldots$ & $\ldots$ & $\ldots$ & 2.2 & 0.006 & 18.19 & 8.65 & K2 & $\mathrm{C}$ \\
\hline ZZ Tau AB & 67.713750 & 24.706111 & 15.24 & 0.031 & 0.023 & $\ldots$ & $\ldots$ & $\ldots$ & 2.1 & 0.016 & 110 & 9.51 & M3 & $\mathrm{M}$ \\
\hline FN Tau & 63.560833 & 28.466111 & 15.33 & 0.032 & 0.023 & $\ldots$ & $\ldots$ & $\ldots$ & 2.4 & 0.023 & 179 & 8.69 & M5 & M \\
\hline TrES J041706+264414 & 64.276250 & 26.737222 & 15.37 & 0.033 & 0.023 & $\ldots$ & $\ldots$ & $\ldots$ & 1.6 & 0.001 & 11.83 & 6.05 & K3 & $\mathrm{C}$ \\
\hline
\end{tabular}




\begin{tabular}{|c|c|c|c|c|c|c|c|c|c|c|c|c|c|c|}
\hline Name & $\begin{array}{l}\text { J2000 } \\
\text { R.A. } \\
\text { (deg) }\end{array}$ & $\begin{array}{l}\text { J2000 } \\
\text { Decl. } \\
\text { (deg) }\end{array}$ & $\begin{array}{c}r \\
\mathrm{Mag} \\
(\mathrm{mag}) \\
\end{array}$ & $\begin{array}{l}\text { White } \\
\text { Noise } \\
(\mathrm{mag})\end{array}$ & $\begin{array}{c}\text { Red } \\
\text { Noise } \\
(\mathrm{mag})\end{array}$ & $\begin{array}{c}\text { Lit. } \\
\text { Period }^{\text {a }} \\
\text { (days) }^{\text {days }}\end{array}$ & $\begin{array}{l}\text { Lit. } \\
\text { Ref }^{\mathrm{a}, \mathrm{b}}\end{array}$ & $\begin{array}{c}\text { TrES } \\
\text { Period } \\
\text { (days) }\end{array}$ & $\begin{array}{l}\text { Per. } \\
\text { Signif. } \\
\left(S_{T}\right)\end{array}$ & $\begin{array}{l}\text { Per. } \\
\text { Ampl. } \\
\text { (mag) }\end{array}$ & $\begin{array}{l}\text { Peak } \\
\text { Power }\end{array}$ & $\begin{array}{c}\text { Power } \\
\sigma\end{array}$ & $\begin{array}{l}\text { Spec. } \\
\text { Type }\end{array}$ & $\begin{array}{c}\text { Member } \\
\text { Status }\end{array}$ \\
\hline 2MASS J04203918+2717317 & 65.164167 & 27.293056 & 15.64 & 0.035 & 0.025 & $\ldots$ & $\ldots$ & $\cdots$ & 2.1 & 0.015 & 60 & 8.71 & $\cdots$ & M \\
\hline FW Tau ABC & 67.371250 & 26.280556 & 15.79 & 0.047 & 0.034 & $\ldots$ & $\ldots$ & $\ldots$ & 1.7 & 0.021 & 70 & 10.99 & M4 & M \\
\hline TrES J041937+265259 & 64.902500 & 26.883056 & 15.88 & 0.056 & 0.040 & $\ldots$ & $\ldots$ & $\ldots$ & 1.0 & 0.022 & 25 & 10.42 & G3 & $\mathrm{C}$ \\
\hline TrES J042136+253835 & 65.398333 & 25.643056 & 15.92 & 0.050 & 0.035 & $\ldots$ & $\ldots$ & $\ldots$ & 1.1 & 0.012 & 9 & 5.51 & F6 & $\mathrm{C}$ \\
\hline V892 Tau & 64.669167 & 28.320556 & 15.97 & 0.046 & 0.033 & .. & $\cdots$ & $\cdots$ & 1.7 & 0.018 & 78 & 7.48 & B8 & M \\
\hline TrES J041160+294237 & 62.998750 & 29.710278 & 16.09 & 0.067 & 0.047 & $\ldots$ & $\ldots$ & $\ldots$ & 1.8 & 0.017 & 14 & 6.97 & G8 & $\mathrm{C}$ \\
\hline TrES J041543+290960 & 63.928333 & 29.166667 & 16.23 & 0.065 & 0.047 & $\ldots$ & $\ldots$ & $\ldots$ & 2.6 & 0.078 & 547 & 7.8 & M0 & M \\
\hline FS Tau Aab & 65.508333 & 26.958611 & 16.39 & 0.090 & 0.067 & $\ldots$ & $\ldots$ & $\ldots$ & 2.2 & 0.084 & 331 & 4.66 & M0 & M \\
\hline CIDA-2 & 63.771667 & 28.145833 & 16.44 & 0.070 & 0.050 & $\ldots$ & $\ldots$ & $\ldots$ & 1.7 & 0.024 & 37 & 6.31 & M4.5 & M \\
\hline TrES J041427+255130 & 63.613750 & 25.858333 & 16.51 & 0.10 & 0.071 & $\ldots$ & $\ldots$ & $\ldots$ & 0.9 & 0.044 & 14 & 5.10 & G5 & $\mathrm{C}$ \\
\hline
\end{tabular}

Notes.

${ }^{a}$ For objects with multiple period measurements in the literature that are functionally identical $(\delta P \leqslant 1 \%)$, we list a single representative period measurement in parentheses, and group the relevant reference codes in parentheses as well. Literature periods adopted in the K-S tests in Sections 4.2 and 4.3 are highlighted in italics. Literature periods listed as -99.0 represent monitoring campaigns which report no detectable periodic modulation in the star's photometry.

${ }^{b}$ Literature reference codes_A: Grankin et al. 2008; B: Bouvier et al. 1995; C: Bouvier et al. 1993; D: Osterloh et al. 1996; E: Percy et al. 2010; F: Norton et al. 2007; G: Grankin 1997; H: Grankin 1993;

I: Stelzer et al. 2003; J: Rydgren et al. 1984; K: Bouvier et al. 1997. Dot means no reference.

${ }^{c}$ TrES period significance $\left(S_{T}<8\right)$, but object promoted to "Definitely Periodic" due to integer agreement between TrES period and literature period.

d Excluded from "Definitely Periodic" classification due to ambiguous period; periodogram contains secondary non-alias peak $>1 / 2 \times$ primary peak height.

e Excluded from "Definitely Periodic" classification as best period appears to be a one-day alias.

${ }^{\mathrm{f}}$ Excluded from "Definitely Periodic" classification because periodic amplitude is less than $2 \times$ amplitude of red noise. 


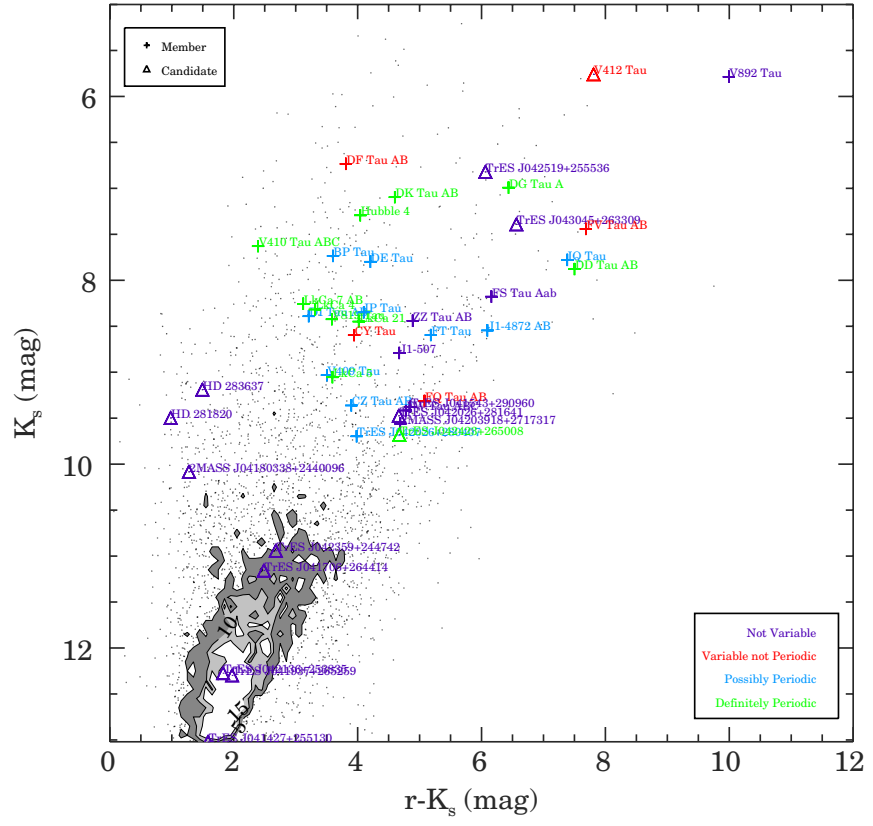

Figure 2. $r-K_{S}$ vs. $K_{S}$ color-magnitude diagram for all TrES/Spitzer detections in the TrES Taurus field. Black dots represent all TrES objects matched with Spitzer, while other symbols highlight the membership status determined for Taurus members and candidates by Rebull et al. (2010). We use contours instead of individual points to indicate the density of non-members in saturated regions of color-magnitude space. The color of each symbol indicates the visual classification of each source's light curve, as described in Section 3.3. Bona fide Taurus members are generally redder and brighter than typical field stars, and also typically exhibit significant photometric variability.

(A color version of this figure is available in the online journal.)

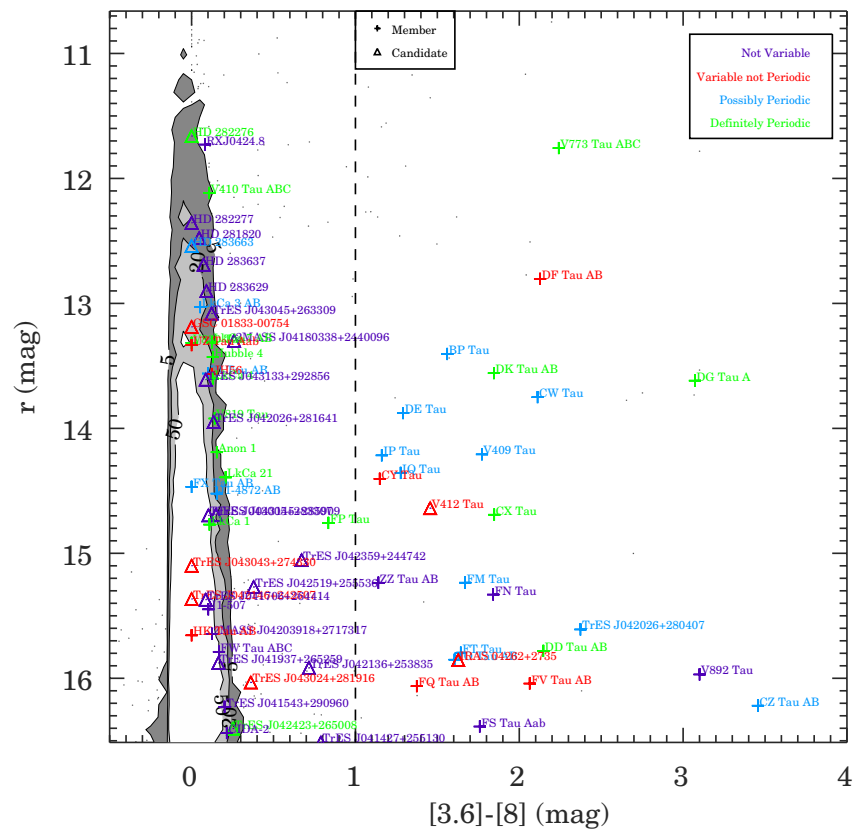

Figure 3. [3.6]-[8] vs. $r$ color-magnitude diagram for all TrES/Spitzer detections in the TrES Taurus field. Symbols and contours are as in Figure 2; the vertical dashed line separates Class II objects (with circumstellar disks, and $[3.6]-[8]>1.0$ ) from Class III objects ([3.6]-[8] $<1.0$, and lacking circumstellar disks). Roughly one-third of the TrES-detected L1495 members show evidence for circumstellar disks; these stars are more likely to exhibit photometric variability than candidate L1495 members that lack circumstellar disks.

(A color version of this figure is available in the online journal.)

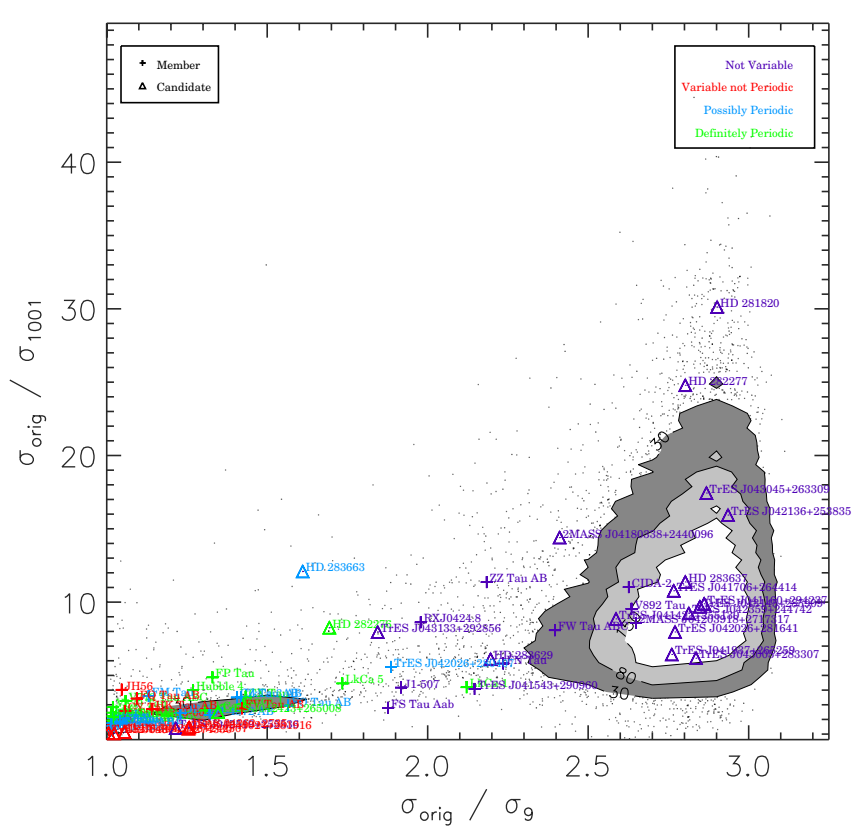

Figure 4. Standard deviation ratio diagram for all TrES/Spitzer detections in the TrES Taurus field. Symbols and contours are as in Figure 2; the horizontal axis depicts the ratio of the original light curve's standard deviation to that obtained after smoothing the light curve with a boxcar window with a width of 9 data points. The vertical axis depicts the ratio of the original light curve's standard deviation to that obtained after smoothing the light curve with a window 1001 data points across. Most Taurus members lie in the lower left corner of the diagram, indicating that their light curves are dominated by temporally structured variability.

(A color version of this figure is available in the online journal.)

and weeks, respectively. TrES light curves are not uniformly sampled, with gaps due to the nightly observing cadence as well as weather effects, but TrES' sampling density is sufficiently high that smoothing over these gaps does not compromise the utility of this technique for identifying variable sources. We plot in Figure 4 the ratios of the standard deviation of the original light curve to the standard deviations of the smoothed light curves. There is a clear concentration of points at $\sigma_{\text {orig. }} / \sigma_{9}=3$ and $\sigma_{\text {orig. }} / \sigma_{1001}=10-20$, representing the vast majority of stars whose TrES light curves have standard deviations that scale as $1 / \sqrt{n}$, and thus appear dominated simply by unstructured photometric noise.

Figure 4 also includes, however, a locus of points extending to $\sigma_{\text {orig. }} / \sigma_{9}=\sigma_{\text {orig. }} / \sigma_{1001}=1$. These represent sources with well-detected photometric variability, where smoothing does not significantly reduce the standard deviation (and thus, the temporal structure) of their light curves. There is a clear concentration near 1,1 , but there is also a continuum of points stretching between the variable and non-variable clumps, representing the smooth continuum between sources with variability timescales and amplitudes that exceed our smoothing window and photometric noise level, respectively, and are thus easily detected as variable sources, and those whose variations have timescales and amplitudes outside our range of detectability.

Figure 4 demonstrates that the vast majority of the 48 confirmed Taurus members lie in the region of Figure 4 dominated by variable sources. Indeed, most of the known Taurus members lie below $\sigma_{\text {orig. }} / \sigma_{9}=1.6$ and $\sigma_{\text {orig. }} / \sigma_{1001}=5$, which we interpret as definitive evidence that these sources do possess enhanced photometric variability, as seen by numerous other investigators. We therefore establish $\sigma_{\text {orig. }} / \sigma_{9}=1.6$ and 
$\sigma_{\text {orig. }} / \sigma_{1001}=5$ as baseline criteria that can be applied to the rest of the TrES light curves to evaluate additional Taurus candidates as bona fide YSOs and Taurus members.

\subsection{Identifying Stellar Periods in the Presence of Astrophysical Red Noise}

\subsubsection{Identifying Candidate Periods}

We use the Lomb-Scargle (LS; Lomb 1976; Scargle 1982) periodogram to search for periodicities within the light curves of known and candidate Taurus members detected by TrES. We attempt to measure the most likely potential period for each object using the algorithm originally developed by Agüeros et al. (2011) to measure rotation periods from the light curves of low-mass members of the Praesepe open cluster. This iterative technique computes the LS periodogram from each source's original (unsmoothed) TrES light curve, searches the periodogram to identify the period with the most power, then phase-folds the light curve on this "best-fit" period and smooths the folded light curve using a box-car window that includes the nearest $10 \%$ of the phased epochs. Computing the residuals between the original light curve and the phase-folded, smoothed version, we eliminate all $4 \sigma$ outliers before calculating a new periodogram from the $\sigma$-clipped (but unfolded, unsmoothed) light curve. We adopt the period with the maximum power in the periodogram computed after three iterations of this process as the candidate rotation period for each source. To illustrate this process, Figure 5 shows the original TrES light curve for V410 Tau ABC, as well as the periodogram and phased light curve produced by the last iteration of our analysis.

To verify that each source's candidate period is astrophysically significant, and not due solely to aliasing effects, we reject all candidate periods with a duration close $( \pm 1.5 \%)$ to 1 day, and flag likely beat periods in each periodogram using the wellknown formula

$$
f_{\text {beat }}=f_{1}-f_{2},
$$

where $f_{1}=1 / P$ and $f_{2}=n /(1$ day $)$, with $|n| \leqslant 5$. As can be seen in the second panel in Figure 5, most of the peaks in V410 Tau ABC's periodogram are beat periods, aside from its maximum likelihood period ( $P=1.869$ days) and the one-day alias (and its integer frequency multiples).

\subsubsection{Statistical Tests for Evaluating the Significance of Candidate Periods}

Even after obvious aliases and beat periods have been excluded, interpreting the statistical significance of structures within a periodogram is a complex problem, particularly for light curves that may contain periodic behavior on multiple timescales and/or aperiodic but highly structured variations. Analytic formalisms have been developed to calculate the false alarm probability (FAP) associated with a peak within a given periodogram, but these FAPs are strictly speaking only applicable to systems where the light curve's uncertainties are dominated by random, temporally uncorrelated measurement errors (commonly referred to as "white noise"; for example analytic FAP formulae, see Scargle 1982; Horne \& Baliunas 1986). Numerous subsequent studies have examined how to best analyze the periodogram to provide optimal sensitivity to bona fide astrophysical periods and to mitigate potential biases due to sampling effects (both in terms of the sampling of the input light curve, and of the distribution of frequencies on which the periodogram is computed). Most recent studies have concluded that Monte Carlo tests using the input light-curve time-stamps, adopted periodogram grid, and either synthetic random noise or temporally scrambled measurements provide the most reliable means of computing accurate and reliable FAP statistics for systems dominated by white noise (e.g., Koen 1990; Cumming et al. 1999; Reegen 2007; Frescura et al. 2008; Sturrock \& Scargle 2010).

A few astrophysical light curves contain only idealized white noise and many include major contributions from temporally correlated signals related to measurement effects (e.g., biases due to slow drifts in weather conditions, airmass, instrumental noise, etc.). The TrES light curves have been detrended to remove most such observational signals, but the $\mathrm{T}$ Tauri star light curves we seek to analyze may, and often do, contain additional temporally correlated variations of an astrophysical nature, due to changes in the T Tauri star's accretion rate or line-of-sight extinction. The presence and ill-defined scales of these temporally correlated signals (commonly referred to as "red noise") violate the conditions that underpin FAPs calculated assuming only white noise, including both analytic and Monte Carlo approaches. Efforts are underway to derive techniques for calculating the FAP for a given periodogram in a way that is robust and reliable even for light curves with a significant red-noise component. These techniques are still developing, however, and are often computationally or mathematically complex (Vio et al. 2010), or have only been tested with particular types of time-series data (e.g., light curves with power-law spectral indices, including X-ray data for active galaxies or stratigraphic geological data; Vaughan 2010; Vaughan et al. 2011).

While a consensus has yet to emerge around a simple method for calculating FAPs that is robust in the presence of red noise for a fully generalized set of light curves, solutions have been found that work well in specific scientific domains. The intense scientific interest in detecting and interpreting planetary transit signals, in particular, has spurred the development of mature methods for calculating FAPs for transit signals in densely populated light curves, such as those we analyze here (e.g., Pont et al. 2006; Southworth 2008; Carter \& Winn 2009). The periodic signal associated with a transit event, which has a typical amplitude at the millimagnitude level and only affects a small fraction of the phase-folded light curve, is notably distinct from the periodic signal associated with the rotational modulation of star spots on pre-main-sequence stars, which occupy the entirety of the phase-folded light curve and can possess amplitudes of several tenths of a magnitude. While these differences in the morphologies of transit and rotation signatures are non-trivial, they are also mathematically well defined, such that the techniques originally developed to estimate FAPs for transit signatures can be adapted to test the veracity of rotation signatures as well. Indeed, in their Figure 9, Pont et al. (2006) show the sensitivity of their FAP estimator to the presence of astrophysical red noise, as well as observational red noise: accounting for the presence of red noise decreases the significance of all transit events, but the largest decreases in significance are associated with candidate transit events within light curves that exhibit obvious sinusoidal modulation signatures (a source of astrophysical red noise for transit detections).

To establish the veracity (or lack thereof) of the candidate periods that we identify in each T Tauri star's periodogram, we have adapted the formalism developed by Pont et al. (2006) to calculate significance levels for candidate transit signatures. 

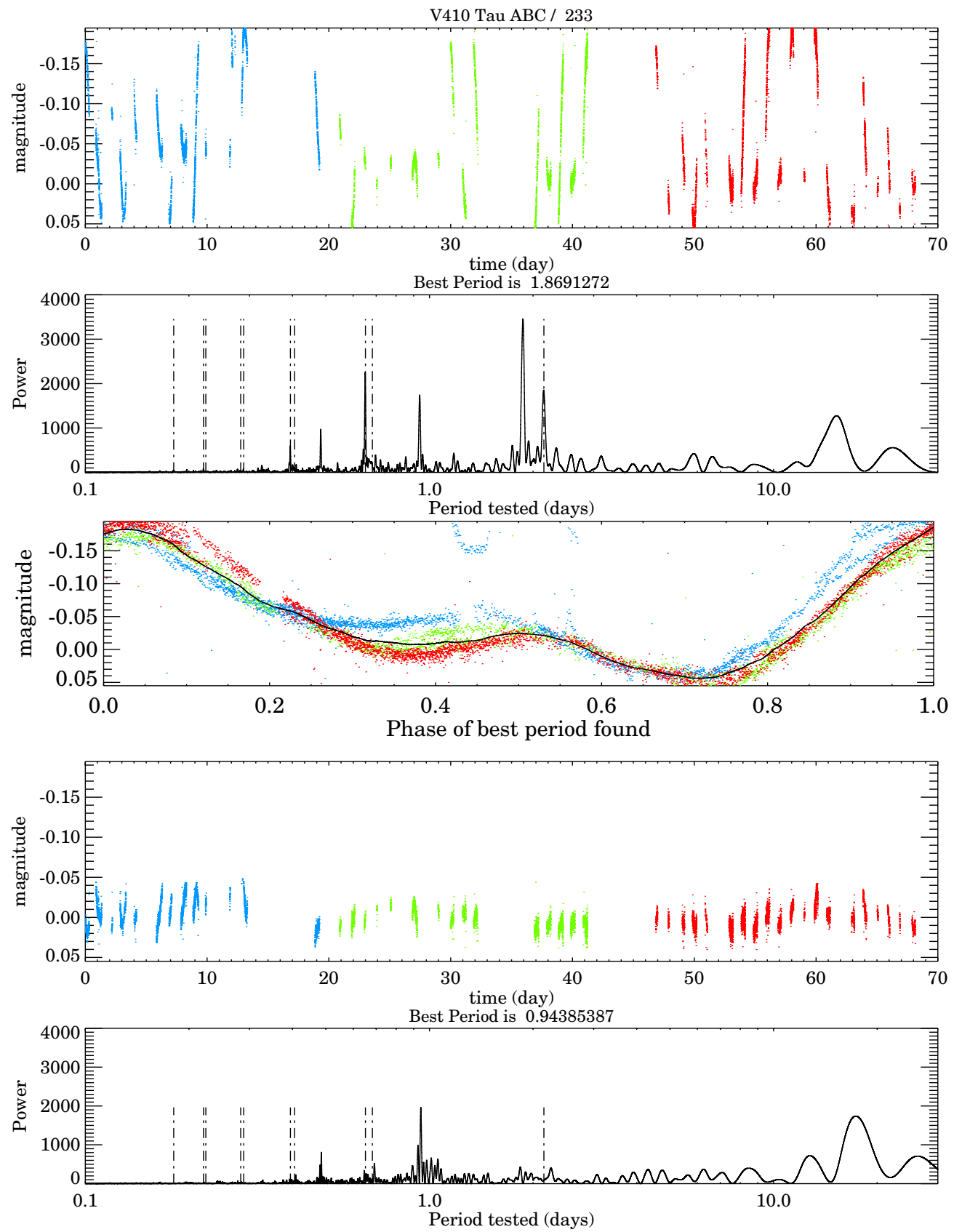

Figure 5. Illustration of our period-finding and pre-whitening procedure, using Taurus member V410 Tau as an example. First panel: the original TrES light curve for V410 Tau. Second panel: the periodogram resulting from our iterative Lomb-Scargle technique, which identifies V410 Tau's most likely period as $\sim 1.87$ days. Dashed vertical lines indicate the first 10 beat periods between the most likely period and a possible one-day alias. Third panel: V410 Tau's light curve, phased to a period of 1.87 days. The black curve is the smoothed version of the phase-folded light curve used to pre-whiten the original light curve. Fourth panel: the pre-whitened LC, with much of the previous temporal structure removed. Fifth panel: the LS periodogram calculated for the pre-whitened LC via our iterative technique. Vertical dashed lines represent the same beat periods as in the second panel. The power at the original best period position has been significantly reduced, as have the associated beat periods, confirming that the period measured for V410 Tau appears to be a genuine astrophysical signal.

(A color version and a complete figure set (74 images) of this figure are available in the online journal.)

In their Section 4, Pont et al. (2006) present an expression for evaluating the significance of a given transit signature:

$$
\frac{d^{2} n^{2}}{\sum_{k=1}^{N_{t r}} n_{k}^{2}\left(\frac{\sigma_{w}^{2}}{n_{k}}+\sigma_{r}^{2}\right)}>S_{T}^{2},
$$

where $d$ represents the depth of the transit signature, $n$ is the number of data points in the full light curve, $N_{k}$ is the number of distinct transit events in the full light curve, $n_{k}$ is the number of data points in the $k$ th transit event, $\sigma_{w}$ is the amplitude of the light curve's white-noise component, $\sigma_{r}$ is the amplitude of the light curve's red-noise component, and $S_{T}$ is the threshold for discriminating significant and non-significant transit events. Pont et al. (2006) identified $S_{T} \sim 10$ as an appropriate significance threshold for most transit events. Pont et al. (2006) also demonstrate that $\sigma_{r}$ and $\sigma_{w}$ can be empirically determined from the dependence of the variance of a binned prewhitened light curve on the number of data points over which the light curve was binned. Indeed, the $\sigma_{\text {orig. }} / \sigma_{9}$ and $\sigma_{\text {orig. }} / \sigma_{1001}$ ratios defined in 3.1 actually represent simple projections of the full behavior of $\sigma_{n}$, and their utility for identifying T Tauri stars 
and other variables reveals that the astrophysical phenomena we seek to identify are actually themselves sources of astrophysical red noise.

To adapt the Pont et al. (2006) formalism to our analysis, we have followed their prescription to measure the whiteand red-noise components within the TrES light curves of each of our candidate Taurus members, and have modified several parameters in Equation (3) to describe the sinusoidal modulation that is the signature of rotation within a star's light curve. Specifically, our modified equation for evaluating the significance of a given candidate period is

$$
\frac{d^{2} n^{2}}{\sum_{k=1}^{N_{P}} n_{p}^{2}\left(\frac{\sigma_{w}^{2}}{n_{p}}+\sigma_{r}^{2}\right)}>S_{T}^{2},
$$

where $n, \sigma_{w}, \sigma_{r}$ and $S_{T}$ represent the same values as in the original Pont et al. (2006) formulation. The meanings of the other parameters are slightly modified from the original Pont et al. (2006) formulation to better describe the continuous variations associated with spot modulation of the light curve. In this implementation, $d$ represents the peak-to-peak amplitude of the periodic modulation, $N_{P}$ the number of distinct periodic modulations within the full light curve, and $n_{p}$ the number of data points in the $k$ th period.

To test if each source's maximum power period represents a genuine astrophysical periodicity, or rather a spurious signal due to a fortuitous arrangement of white/red noise, we calculate a significance parameter for each light curve following Equation (4). To assess the level of white and red noise in each light curve, we follow the prescription of Pont et al. (2006) in measuring $\sigma_{w}$ and $\sigma_{r}$ from a "pre-whitened" version of each light curve. We pre-whiten each light curve by subtracting a copy of the light curve that has been phase-folded to the period with the maximum power in the periodogram and smoothed by a 401-epoch boxcar function, as demonstrated in Figure 5. This pre-whitening procedure removes the structure in the light curve that is associated with the candidate periodicity, but preserves all other red-noise signals in the light curve, either astrophysical or observational in nature. After returning the pre-whitened light curve to its original, un-phase-folded temporal axis, we then follow Pont et al. (2006) in measuring $\sigma_{w}$ and $\sigma_{r}$ from each prewhitened light curve; we also repeat our iterative Lomb-Scargle analysis on the pre-whitened light curve to reveal the amount of structure remaining in the periodogram after subtracting the candidate periodic modulation.

\subsubsection{Identifying Significance Thresholds via Monte Carlo Simulations}

Given the modifications we have made to the Pont et al. (2006) formalism, we have used a Monte Carlo simulation incorporating actual TrES data to determine the $S_{T}$ threshold that corresponds to a reliable period detection. To provide "ground-truth" for this inference, we added synthetic white and red noise to the TrES light curve of $\mathrm{LkCa} 7$, whose rotation period has been well measured by numerous authors (e.g., Grankin 1992; Grankin et al. 2008), and whose TrES light curve exhibits clear rotational modulation. Using the TrES LkCa 7 light curve as a template ensures that our simulated light curves accurately capture the morphology of rotational modulation within a T Tauri star's light curve, and the actual sampling of the TrES observations. We degrade the rotational signature in the $\mathrm{LkCa} 7$ light curve with simulated white noise, adding a random offset to each data point (drawn from a Gaussian with $\sigma=0.0175 \mathrm{mag}$ ), as well as synthetic red noise, which we simulate as a random walk that spans the full length of the LkCa 7 light curve. Specifically, each simulation generates an independent "random-walk" light curve as the cumulative sum of random Gaussian offsets, sampled on a regular 0.25 day grid spanning the full duration of the $\mathrm{LkCa} 7$ light curve (i.e., assuming that the red noise in each T Tauri star light curve is dominated by astrophysical, not observational, effects, this random-walk curve has no gaps due to weather or the day/night cycle). After linearly interpolating the coarsely sampled random-walk light curve onto the more densely sampled TrES data, we added the smoothly varying random-walk component to each data point in the LkCa 7 light curve, introducing a synthetic red-noise component into each degraded $\mathrm{LkCa} 7$ light curve.

Applying our period measurement and significance evaluation codes to a suite of 150 synthetically degraded TrES LkCa 7 light curves, we have identified a threshold for the significance parameter in Equation (4), as well as two other light curve/periodogram parameters, that together indicate a reliable and accurate period measurement. To infer these parameters, we examined the accuracy of the candidate periods determined for $150 \mathrm{TrES}$ LkCa 7 light curves that had been degraded by 0.0175 mag of synthetic white noise, and varying levels of red noise to sample the full range of $\sigma_{r}$ values $\left(0.0<\sigma_{r}<0.2\right)$ measured from TrES T Tauri star light curves. Specifically, we generated 10 degraded copies of the LkCa 7 light curves at each of 15 different levels of additional artificial red noise, producing 150 light curves with measured $\sigma_{r}$ values distributed evenly between 0.0 and 0.4 , corresponding to twice the range of $\sigma_{r}$ values measured for the TrES T Tauri star light curves. We then used our periodogram analysis routines to measure a candidate period for each light curve, and calculated the significance parameter from Equation (4), as well as two additional ratios we identified as useful for evaluating period measurements: the ratio of the first and second strongest (non-alias) peaks in the periodogram, and the ratio of the candidate periodic modulation to the amplitude of the red noise measured in the pre-whitened light curve. The distributions of these parameters are shown in Figure 6 for all 150 light curves, with symbols identifying the parameters associated with successful measurements of LkCa 7's period (i.e., $5.5<P<5.75$, corresponding to $\delta P<2 \%$ ). The global success rate for recovering $\mathrm{LkCa} 7$ 's period from the degraded light curves was only marginally better than $50 \%$ (79/150), but adopting the significance thresholds shown in Figure 6 isolates a sample of 70 high-confidence rotators with an $\sim 85 \%$ (59/70) success rate for accurately recovering LkCa 7's period. As Figure 6 indicates, the significance parameter is the most useful metric for identifying accurate period measurements, with a threshold value of 8 providing a good discrimination between accurate and inaccurate period measurements. The ratio of the strengths of the primary and secondary (nonalias) periodogram peaks also proves to be a useful indicator of an accurate period measurement: accurate period measurements are preferentially found at low secondary-to-primary ratios, indicating the presence of a strong, unambiguous signal in the periodogram. Periodograms with primary-to-secondary ratios closer to one, by contrast, are preferentially dominated by erroneous period measurements, revealing the difficulty of extracting accurate period measurements from periodograms with multiple strong peaks.

As a last check, we also calculate a traditional FAP threshold to evaluate the likelihood that the observed periodogram power could arise from the white and red noise within the light curves 


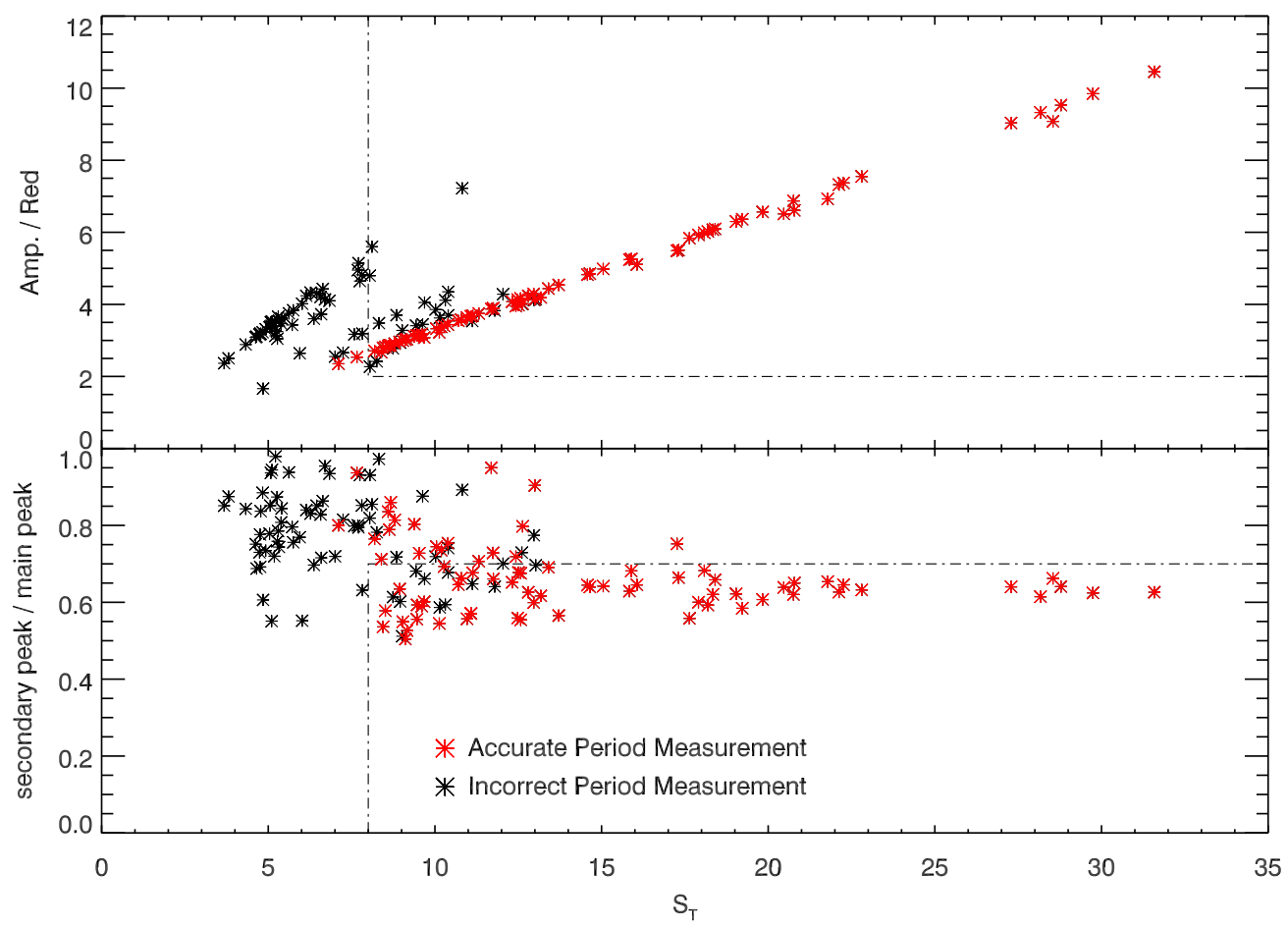

Figure 6. Significance parameters calculated for 150 copies of the LkCa 7 TrES light curve after degrading the light curve by additional white and red noise, plotted against additional statistics useful for discriminating accurate and inaccurate period measurements: the ratio of the amplitudes of the candidate periodic modulation to the measured red-noise level (top panel), and the ratio of the primary and secondary (non-alias) periodogram peaks (bottom panel). Dashed lines indicate thresholds in each parameter that discriminate between simulated light curves whose periods were and were not correctly identified (red and black symbols, respectively). Before utilizing the reliability indicators shown above, the raw success rate for successfully extracting LkCa 7's period from these artificially degraded light curves was only slightly larger than $50 \%$; that is, 79 of the 150 artificially degraded light curves produced periodograms where the strongest peak was within $2 \%$ of LkCa 7's actual period. Implementing the reliability thresholds shown above (i.e., $S_{T}>8$, Amp./Red $>2$, and secondary peak/main peak $<0.7$ ), however, generates a much higher fidelity sample: of the 70 light curves that met these criteria, 59 produced candidate period measurements that correctly identified LkCa 7 's underlying period, corresponding to an $85 \%$ success rate. Guided by these simulations, we use similarly conservative thresholds in each of these parameters to assess the reliability of the candidate period measured from each TrES light curve.

(A color version of this figure is available in the online journal.)

of other non-T Tauri stars in this TrES field. As noted above, this FAP will inevitably underestimate the true FAP given the presence of substantial astrophysical sources of red noise in these T Tauri star light curves (i.e., aperiodic variability due to accretion variations). Nonetheless, a traditional FAP estimate can provide a useful relative indication of the strength of the variability associated with each T Tauri star's candidate period. To generate an FAP estimator that is maximally reflective of the white- and red-noise content of the TrES observations, we examined the distribution of periodogram peak heights produced by our analysis pipeline for the pre-whitened light curves of 942 stars with $\sigma_{\text {orig }} / \sigma_{9} \leqslant 2.0$. Selecting stars with $\sigma_{\text {orig }} / \sigma_{9} \leqslant 2.0$ helps ensure that the resultant FAP threshold is a conservative threshold, as these stars' original light curves appear to show some evidence for elevated variability content, either due to time-correlated errors or intrinsic source variability. The prewhitening process will remove the power associated with the dominant periodic signal within the light curve, but the power at all other frequencies will remain, preserving any other timecorrelated errors that may be present in the light curve, such as those due to non-periodic changes in the seeing or atmospheric transparency, for example. Visual inspection of all 942 prewhitened light curves verified that none of the pre-whitened light curves showed evidence for intrinsic source variations, suggesting that the dominant temporal structure is associated with the error terms.

The distribution of maximum peak heights measured from the periodograms of these non-variable sources are shown in
Figure 7; from this distribution, we infer that periodogram peaks with powers $>1150$ and 2300 indicate $95 \%$ and $99 \%$ probabilities, respectively, of diagnosing genuine astrophysical variations (which may nonetheless simply trace aperiodic variability for our T Tauri star targets). To evaluate the significance of the variability identified for each known or candidate Taurus member, we show in Figure 8 the maximum power in each object's periodogram, with the 95\% and 99\% FAP thresholds indicated for comparison. This plot also indicates the significance of each star's largest periodogram peak after normalizing by the rms of the full power distribution within the periodogram, providing another check on the significance of the periodogram peak. Table 1 summarizes the statistical properties of each star's light curve and periodogram, including the light curve's standard deviation, median error, and white $\left(\sigma_{w}\right)$ and red $\left(\sigma_{r}\right)$ noise content, the significance parameter $\left(S_{T}\right)$ and amplitude associated with the candidate periodic modulation, as well as the maximum peak height and global rms of the star's periodogram.

\subsection{Classifying Periodic and Aperiodic Variables}

Using the statistical parameters identified above as useful indicators for the reliability of a given period measurement, as well as a visual inspection of each star's light curve, we classified the 74 Taurus members and candidates with TrES light curves into four categories: "Not Detectably Variable," "Variable but Not Periodic," "Possibly Periodic," and "Definitely Periodic." The primary factors in our classification are the numerical parameters provided above, namely, the significance parameter 


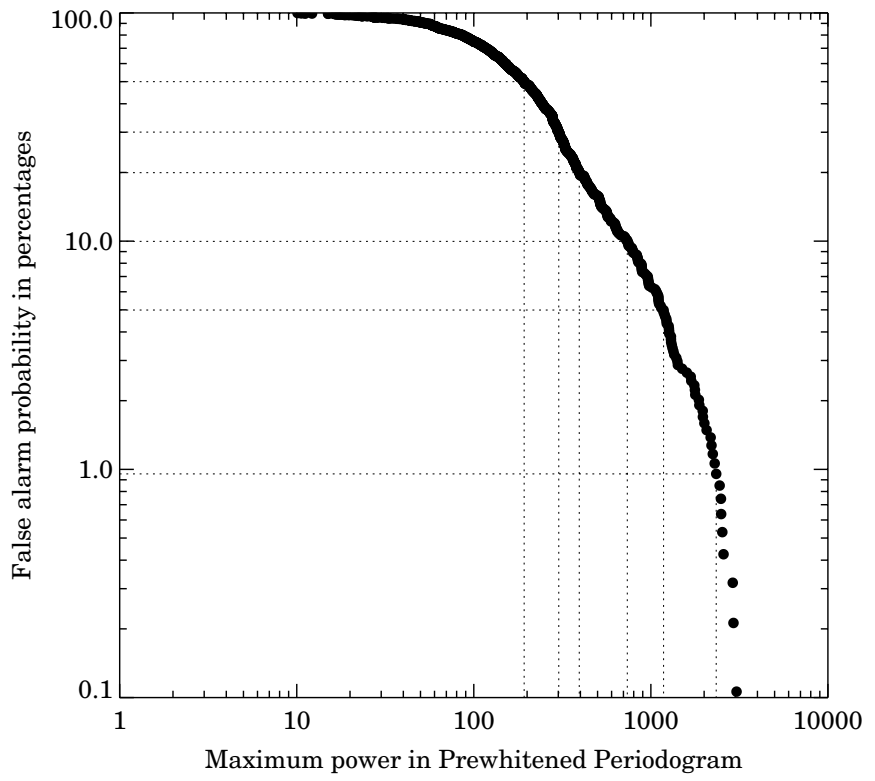

Figure 7. False alarm probability as a function of periodogram peak power, estimated empirically by computing periodograms from pre-whitened light curves for 942 potentially variable sources (standard deviation ratio $<2.0$ ). Dashed lines identify periodogram power thresholds that correspond to various false alarm probabilities: $99 \%$ and $95 \%$ probabilities correspond to peak periodogram powers of 2300 and 1150 , respectively.

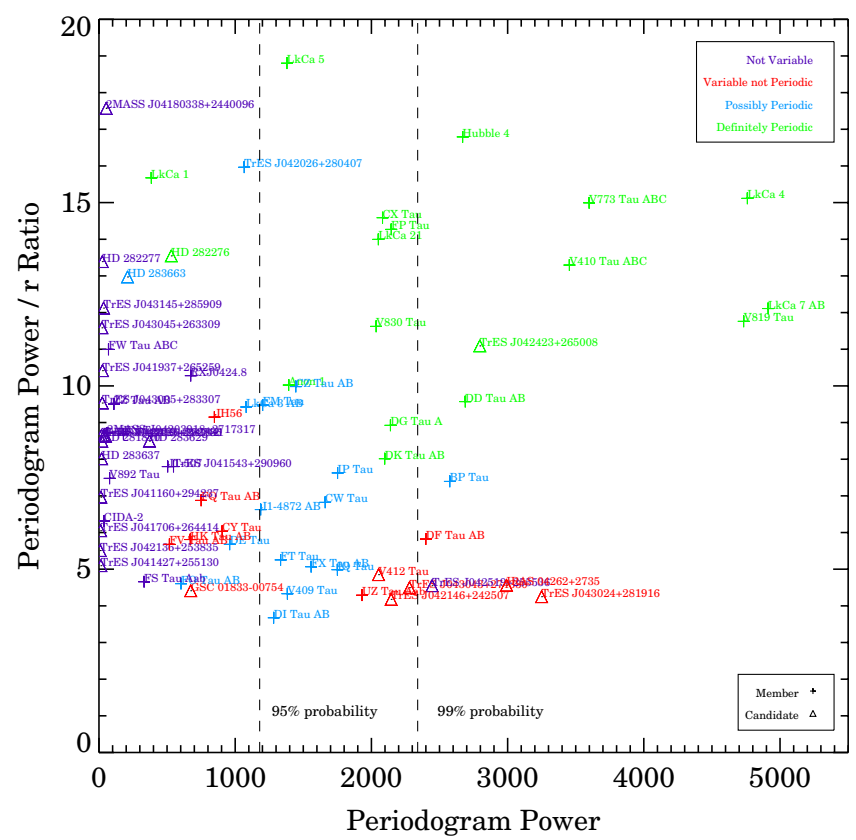

Figure 8. Periodogram power, normalized and unnormalized by the periodogram rms, for all Taurus members. The horizontal axis represents the highest power in the original periodogram with the vertical axis giving the periodogram power after normalizing by the rms of the periodogram. The $95 \%$ and $99 \%$ probability confidence levels for detecting periodic signals based on our empirical false alarm function are indicated by the vertical dashed lines. All but one source with a peak periodogram power $>1150$ is classified as variable; of these sources, those with peak periodogram powers $>6$ times the rms of the periodogram are nearly all classified as "Definitely Periodic" or "Possibly Periodic," while those with peak powers $<6$ times the rms are typically classified as "Variable, Not Periodic."

(A color version of this figure is available in the online journal.)

from Equation (4), the level of white and red noise in each light curve, and the amplitude and periodogram peak ratios

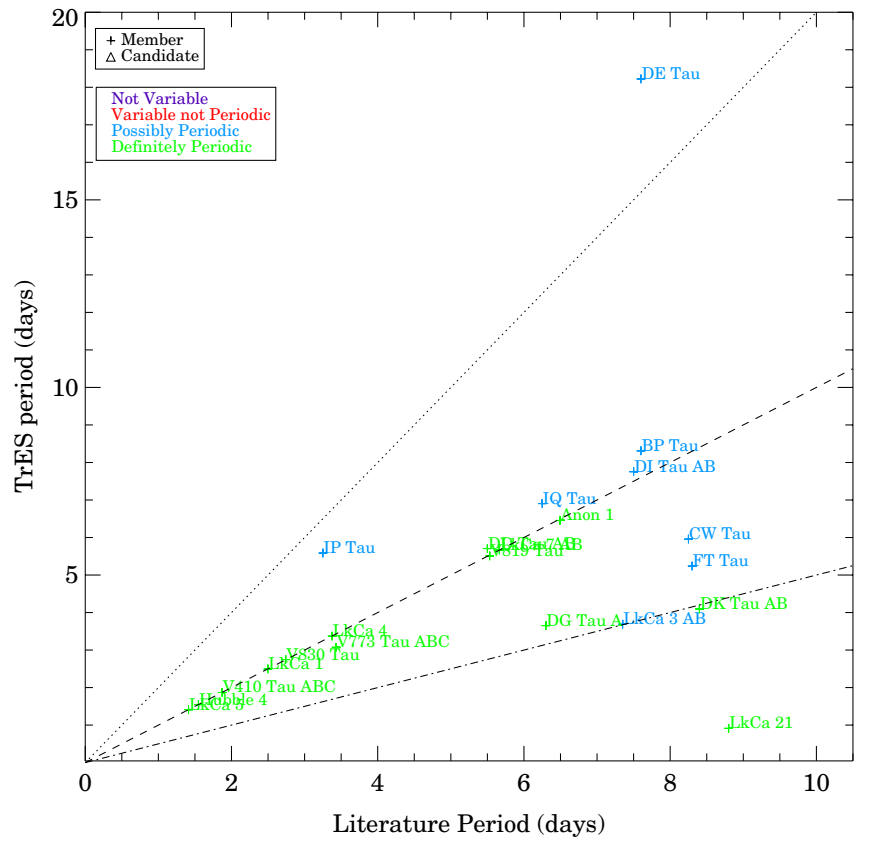

Figure 9. Comparison of the periods we measure from TrES light curves with those reported in the literature for the same objects. The dashed line indicates the loci of perfect agreement between the TrES and literature period; dotted and dash-dotted lines indicate loci where the TrES period is twice and half that reported in the literature, respectively. The periods we measure from the TrES light curves typically agree well with prior determinations; in cases where the periods do disagree, TrES typically finds a shorter period, as expected given the dense sampling of the high-cadence TrES light curves.

(A color version of this figure is available in the online journal.)

shown in Figure 6. We also considered the level of agreement with previously reported period measurements in assessing the reliability of our period measurements; the specific criteria for inclusion within each category are described below. We list the properties of each source's light curve in Table 1, using tiers to group all the sources by classification; we also show light curves for each source in Figure 5, and color-code each source's symbol in Figures 2, 3, 4, 8, and 9 to reflect its final classification.

\subsection{1. "Definitely Periodic" Sources}

We classify $24 \%$ (18/74) of the Taurus members/candidates in our sample as "Definitely Periodic." We include 15 sources in this category whose TrES light curves reveal periodic modulation that is significant $\left(S_{T}>8\right.$ and amp. $\left.>2 \sigma_{r}\right)$ and unambiguous (period is not a 1 day alias [i.e., $0.985<P<1.015$ ], and primary periodogram peak $>2 \times$ secondary (non-alias) periodogram peak). We also include in this category three additional sources (Anon 1, $\mathrm{LkCa} 5$, and $\mathrm{LkCa} 1$ ) whose candidate TrES periods fail the significance threshold adopted above, but the periodogram we calculate for these stars contains a single strong peak at a period corresponding to one previously reported in the literature for these stars, providing independent evidence for the significance of these periodic modulations.

In total, we identify periods previously reported in the literature for 14 of the stars that we classify as "Definitely Periodic." For 11 of these stars, the period we measure from the TrES light curve is identical $(\delta P<1 \%)$ to one previously reported in the literature, supporting the overall reliability of our period measurements. For two additional stars, the discrepancy between the TrES and literature periods can be attributed to aliasing effects: the periods previously reported 
for $\mathrm{DK}$ Tau $\mathrm{AB}$, the more straightforward case, are simply double that inferred from the TrES light curve (TrES period $=$ 4.1 days; literature periods $=8.2$ and 8.4 days). By contrast, the TrES light curve for LkCa 21 phases cleanly on a period of 0.91 days, a significantly shorter period than the 8.7 day period measured by Bouvier et al. (1993) and Percy et al. (2010). The monitoring data analyzed by Bouvier et al. (1993) and Percy et al. (2010) were obtained on a nightly cadence, however, and are thus insensitive to sub-day periods; we therefore attribute the previous $\sim 8.7$ day period measurements to the beat period produced by the nightly sampling frequency and true 0.9 day period. Further support for this sub-day period can be drawn from spectroscopic observations, which also identify LkCa 21 as a rapid rotator ( $v \sin i \sim 46 \mathrm{~km} \mathrm{~s}^{-1}$; Nguyen et al. 2009). Indeed, DG Tau A appears to be the only case where the period measured from the TrES light curve cannot be easily reconciled with those previously reported in the literature (TrES period $=3.65$ days; literature period $=6.3$ days). Appropriately, DG Tau A's candidate period lies quite close to our detection thresholds for the significance parameter $\left(S_{T}=8.1\right)$ and for an unambiguous periodogram peak (primary/secondary $\sim 2.3$ ), indicating this periodicity is among our least confident detections.

We are unable to locate previous period measurements for 4 of the 18 Taurus members/candidates that we classify as "Definitely Periodic." Two of these sources are confirmed Taurus members (FP Tau and CX Tau), while two are candidate members identified by Rebull et al. (2010) (HD 282276 and TrES J042423+265008). Three of the four newly identified periods lie between 2 and 3.5 days, a timescale that has been difficult to probe in previous monitoring programs conducted on a $\sim$ nightly cadence, but which is easily accessed with the densely sampled TrES light curves.

\subsection{2. "Variable, Possibly Periodic" and "Variable, Not Periodic" Sources}

We classify $42 \%(31 / 74)$ of the Taurus members and candidates as either "Variable, Possibly Periodic" or "Variable, Not Periodic" during the TrES monitoring campaign. These sources exhibit moderate levels of photometric variability $\left(S_{T}>4\right.$, amp. $>2 \times \sigma_{r}$ ); many show significant levels of variability $\left(S_{T}>8\right)$ but failed the secondary criteria for inclusion in the "Definitely Periodic" category (i.e., the source's primary/ secondary periodogram peak ratio $<2$, or the candidate period is an obvious 1 day alias).

Based on a subjective visual examination of the source's light curves and periodograms, we sub-divide these 31 "Variable" sources into 15 "Variable, Possibly Periodic" and 16 "Variable, Not Periodic" sources. As Table 1 and Figure 8 indicate, however, these subjective classifications do correlate with objective parameters: the "Possibly Periodic" sources tend to have periodogram peaks that are weaker in terms of their absolute power, but stronger relative to the rms of the full periodogram.

The enhanced peak/background contrast in the periodograms of the "Possibly Periodic" sources is also evident in the prevalence of existing period measurements for these two classes of sources: we have identified period measurements in the literature for more than half $(8 / 15)$ of the "Possibly Periodic" sources, but for only a quarter $(4 / 16)$ of the sources we classify as "Variable, Not Periodic." The more modest reliability of the periods measured for these sources, however, can also be seen by comparing their existing period measurements to those inferred from the TrES light curves: as shown in Figure 9, only three of the eight sources that we classify as "Possibly Periodic" have
Table 2

Classification of Taurus Members/Candidates

\begin{tabular}{lccccc}
\hline \hline Classification & $\begin{array}{c}\text { Not } \\
\text { Variable }\end{array}$ & $\begin{array}{c}\text { Variable } \\
\text { Not Periodic }\end{array}$ & $\begin{array}{c}\text { Possibly } \\
\text { Periodic }\end{array}$ & $\begin{array}{c}\text { Definitely } \\
\text { Periodic }\end{array}$ & Total \\
\hline Confirmed Members & 9 & 9 & 14 & 16 & 48 \\
Candidate Members & 16 & 7 & 1 & 2 & 26 \\
Total & 25 & 16 & 15 & 18 & 74 \\
\hline
\end{tabular}

TrES and literature periods that agree to better than $\sim 10 \%$ (BP Tau, DI Tau AB, and IQ Tau). Of the five discrepancies, only one (LkCa3 AB) appears to be an obvious alias; the remainder $(\mathrm{CW}$ Tau, FT Tau, IP Tau, and DE Tau) show discrepancies between the two measured periods of at least 0.5 days.

It is also interesting to note that the periods measured for "Possibly Periodic" sources, both those measured here as well as those reported in the literature, appear to be biased toward longer periods than those measured for the stars that we classify as "Definitely Periodic." This may partially reflect that the $\sim 60$ day TrES monitoring window provides better leverage for identifying shorter period systems, but 8 day periods would still seem to be within the reach of a 60 day monitoring program. Another possibility is that longer period T Tauri stars exhibit higher levels of aperiodic variability, resulting in less reliable/accurate period measurements. We will return to this possibility in Section 4.3, when we interpret our period measurements in the context of angular momentum evolution of pre-main-sequence stars.

Notable within the "Variable, Not Periodic" group are the four systems for which periods were previously reported in the literature: DF Tau AB, UZ Tau Aab, JH 56, and CY Tau. These sources are clearly variable, but the phased light curves do not phase cleanly at the reported period, nor do the periodograms contain a strong peak at the previously reported period. The periodograms do reveal other moderately strong peaks (power 800-2000), but the light curves do not phase cleanly in these periods either, either because the periodogram peak is spurious, or the periodic modulation could be diluted by contemporaneous non-periodic variability. Similarly transient period detections have been seen been before in other surveys of star-forming regions; Rebull et al. (2000) found that the periods identified in their first season's monitoring data were only recovered for half of their sources in their second season of monitoring. As we are unable to confirm the prior period measurements for these sources, or provide a reliable alternative period measurement, we classify these sources as "Variable, Not Periodic" during the time span of the TrES monitoring.

\subsection{3. "Not Detectably Variable" Sources}

We classify the remaining 34\% (25/74) of the Taurus members and candidates as "Not Detectably Variable" during the TrES monitoring campaign. These stars (largely candidate, not known, members of Taurus; we analyze variability as a membership indicator in further detail in Section 4.1) have light curves consistent with unstructured white noise $\left(S_{T}<4\right.$ and $\left.\sigma_{r} \lesssim \sigma_{w}\right)$. Consistent with this classification, these stars also exhibit low periodogram powers (typically $<100$; see Figure 4).

\section{RESULTS}

\subsection{Variability as Membership Indicator}

As Table 2 summarizes, we detect clear signatures of photometric variability from more than $80 \%$ (39/48) of the confirmed Taurus members in the TrES footprint; the vast majority of the 
Table 3

Taurus Sources Not in TrES Sample

\begin{tabular}{lccc}
\hline \hline Name & $\begin{array}{c}\text { Spectral } \\
\text { Type }\end{array}$ & $\begin{array}{c}\text { Literature } \\
\text { Period }\end{array}$ & $\begin{array}{r}\text { Literature } \\
\text { Reference }\end{array}$ \\
\hline RY Tau & F8 & 24 & C \\
HD 283572 & G5 & 1.546 & A \\
DH Tau AB & M0.5 & 7.2 & B \\
GI Tau & K5 & 7.2 & B \\
GK Tau & K7 & 4.65 & B \\
DL Tau & G & 9.4 & L \\
\hline
\end{tabular}

Notes. Literature reference codes_A: Grankin et al. 2008; B: Bouvier et al. 1995; C: Bouvier et al. 1993; L: Kundurthy et al. 2006.

field stars in the TrES footprint, by contrast, show low levels of variability. As a result, variability information extracted from the TrES light curves can be used to inform the membership status of the candidate Taurus members identified by Rebull et al. (2010). Many of these potential Taurus objects lie near the locus of non-variable objects in Figure 4, suggesting they may be members of the field star population rather than bona fide Taurus members. Other candidate members, such as TrES $042423+265008$, lie near the variable locus in Figure 4 and have periodograms with strong and distinct peaks, such that their variability reinforces their potential as candidate Taurus members. Of the 26 Taurus candidates that lie within the TrES footprint and were classified as a "Possible Member" or "Pending Investigation" by Rebull et al. (2010), 10 appear to exhibit significant variability: HD 282276, HD 283663, GSC 01833-00754, V412 Tau, IRAS 04262+2735, TrES 042146+242507, TrES 042423+265008, TrES 042519+255536, TrES 043024+281916, and TrES 043043+274330. They represent promising targets for additional spectroscopic follow-up to determine their membership status.

\subsection{Taurus Rotation Periods versus Orion and NGC 2264}

The Taurus-Auriga Association's age has been estimated as 1-3 Myr (Kenyon et al. 2008), comparable to the ages of the Orion Nebular Cluster (ONC), NGC 2264, and NGC 6530, three star formation regions that have been targeted by focused photometric monitoring campaigns for the express purpose of cataloging their members' rotation periods. Cieza \& Baliber (2007) studied the rotation periods of "high-mass" stars in both the ONC and NGC 2264; their sample included 144 stars in NGC 2264 with $(R-I)<1.3$ and Spitzer 3.6 and $8.0 \mu \mathrm{m}$ detections, and 133 stars in the ONC with spectral types of M2 or earlier. The distributions of rotation periods measured in the ONC and NGC 2264 span a similar range, with periods from 0.5 to $\sim 34$ days, but the majority of stars in both clusters rotate with periods of 8 days or less. As Cieza \& Baliber (2007) note, there are substantive differences in the two cluster's period distributions: the ONC period distribution appears more clearly bimodal, with peaks at $\sim 1-2$ and 8 days, while NGC 2264's period distribution is more unimodal, with a broader peak at periods of 2-3 days. More recently, Henderson \& Stassun (2012) analyzed optical photometry from a 35 day campaign of NGC 6530 and measured rotation periods for 290 cluster members; the period distribution they measure is relatively flat between 0.5 and 9 days, with only a modest concentration of periods between 1 and 2 days. Henderson \& Stassun (2012) did find a correlation between stellar mass and rotation period in their sample, however, such that the periods measured for stars with $M>0.5 M_{\odot}$ are skewed toward shorter periods, with a
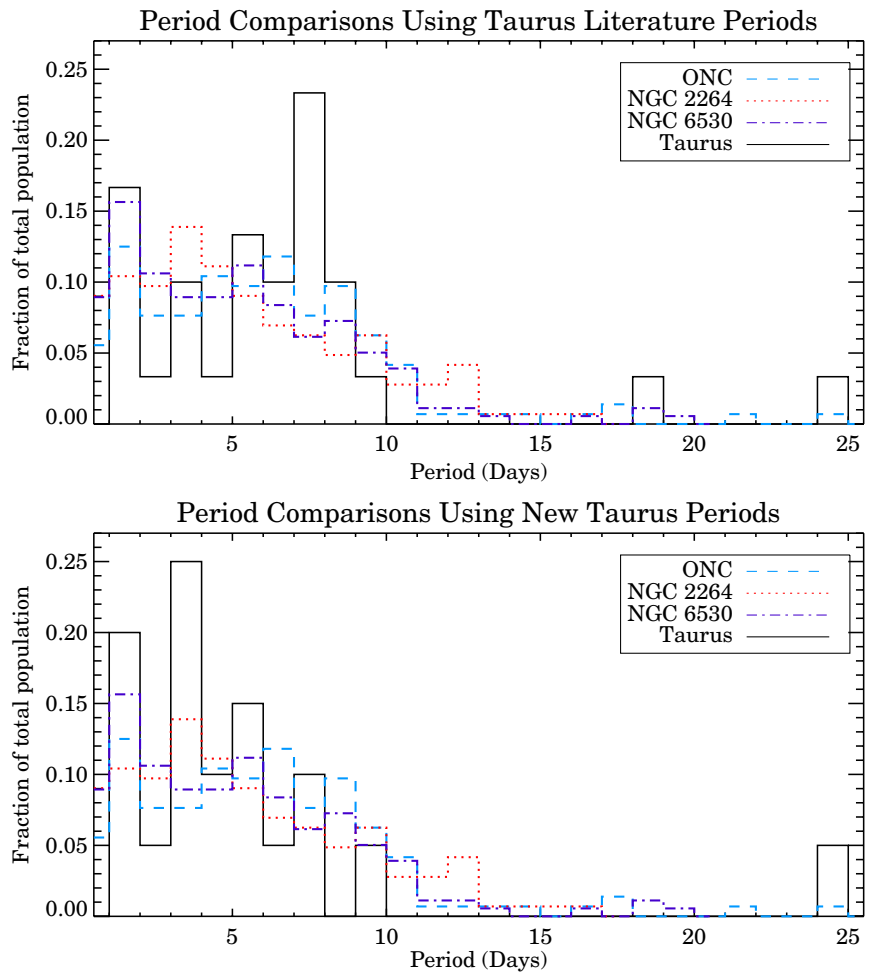

Figure 10. Period distributions of high-mass Taurus members in the TrES L1495 field compared with periods measured for high-mass members of NGC 2264, NGC 6530, and the ONC, three other star-forming regions of similar age. Top: period distributions assuming periods reported in the literature. Bottom: period distributions where TrES periods are adopted for all stars that we classify as "Definitely Periodic", and where periods that are not recovered by TrES are eliminated. After incorporating the TrES period measurements, the period distribution measured for stars in L1495 agrees better with those measured in the ONC, NGC 2264, and NGC 6530.

(A color version of this figure is available in the online journal.)

more prominent excess of 1-2 day periods, and a notable deficit of periods longer than 7 days.

The period distribution for young stars in Taurus, by contrast, has historically featured a larger fraction of slow rotators than seen in other young clusters like the ONC, NGC 2264, or NGC 6530 . This can be seen visually in, e.g., Figure 4 by Rebull et al. (2004), but to demonstrate the statistical significance of the differences between the rotation period distributions seen in Taurus and other young clusters, we construct a sample of rotation periods previously reported in the literature for "highmass" young stars in Taurus; this sample includes rotation periods from the "Lit. Period" column of Table 1 for 24 stars with spectral types of M2 or earlier (adopted periods are shown in italics), and the periods listed in Table 3 for $6 \mathrm{~T}$ Tauri stars in the L1495 field for which, due either to saturation or the presence of nearby neighbors, TrES was not able to obtain reliable light curves. The distribution of periods previously reported in the literature for these Taurus members is shown in the top panel of Figure 10, along with the ONC and NGC 2264 samples compiled by Cieza \& Baliber (2007), and the NGC 6530 sample measured by Henderson \& Stassun (2012): there is a notable deficit of short-period rotators in the Taurus sample (or, equivalently, an excess of long-period rotators) relative to what is seen in these other clusters.

Updating Taurus' period distribution to reflect our classifications from the TrES light curves helps bring the distribution of rotation periods seen in Taurus into considerably better 
agreement with those seen in these other young clusters. We construct a "TrES-based" sample of rotation periods from those measured for 14 high-mass (i.e., spectral types of M2.5 or earlier) Taurus members classified as "Definitely Periodic" in Table 1; we supplement this sample with the six periods reported in Table 3 for stars in the L1495 field but which were not detected by TrES. The period distribution for young stars in Taurus based on our TrES period measurements is shown in the lower panel of Figure 10, and appears significantly closer to those that have been measured for NGC 2264 and the ONC. To quantify the heightened similarity between Taurus' measured period distribution and those seen in other young clusters, we performed a series of two-sided Kolmogorov-Smirnov (K-S) tests between the period distributions measured for Taurus, before and after adopting the TrES rotation periods, and the period distributions measured by others within the ONC, NGC 2264 , and NGC 6530. Adopting the TrES periods for high-mass stars in Taurus increases the probability that Taurus and NGC 2264 share the same parent period distribution from $5 \%$ to $45 \%$; the agreement between the rotation period distributions seen in Taurus and NGC 6530 improve even more, with the likelihood that the two clusters share the same parent distribution increasing from $4 \%$ to $65 \%$. The improved agreement between Taurus, NGC 2264, and NGC 6530 reflects in part the unimodal nature of each of these cluster's period distributions, as well as the effect of excluding from consideration those Taurus members with longer periods reported in the literature, but whose TrES light curves only yield a "Possibly Periodic" classification: of the eight Taurus members whose literature rotation periods we remove from consideration due to their TrES classification as only "Possibly Periodic," seven are reported in the literature as exhibiting periods longer than 6 days. Removing these less reliable, but preferentially longer, periods from Taurus' rotation period sample, we significantly diminish the prevalence of slow rotators in Taurus, and thus increase the agreement between Taurus' period distribution and those seen in other young clusters. The reverse is true in the ONC, however: the ONC's bimodal period distribution includes a substantial number of slow rotators, so removing those stars and adding several shorter period sources to the sample of Taurus rotators decreases the likelihood that Taurus and the ONC share a parent rotation period distribution from $63 \%$ to $23 \%$.

\subsection{Presence of Disks and Angular Momentum Evolution}

Since the earliest measurements of the rotational velocities of $\mathrm{T}$ Tauri stars revealed that they spin well below their break-up velocities (e.g., Vogel \& Kuhi 1981; Hartmann et al. 1986), it has been clear that angular momentum loss plays an important role in the star formation process. To maintain a rotation rate below break-up, $\mathrm{T}$ Tauri stars must actively shed angular momentum as they contract toward the main sequence: otherwise, conservation of angular momentum will cause them to spin up as their radius shrinks, eventually bringing them to their break-up velocity and halting further collapse until some angular momentum could be lost. The exact mechanism for this angular momentum loss is not fully clear, however; while the pre-main-sequence star's magnetic field is almost certainly integral to the process, theoretical descriptions of star-disk interactions (Shu et al. 1994; Hartmann 2002; Mohanty \& Shu 2008), collimated outflows (Shang et al. 2006), and accretionpowered stellar winds (Matt \& Pudritz 2005; Cranmer 2008) have all been proposed to explain this phenomenon.
The mechanisms proposed to explain angular momentum loss in the $\mathrm{T}$ Tauri phase differ in many ways, but they share a common assumption: that angular momentum loss is intimately related to pre-main-sequence mass accretion, and thus to the presence of a substantial circumstellar disk. Circumstellar disks represent the fundamental mass reservoir for the accretion process, so a zeroth-order prediction of the connection between accretion and angular momentum loss then becomes that stars with massive circumstellar disks should rotate more slowly than those stars whose disks have already dissipated: lacking a reservoir to accrete from, these diskless stars will also be unable to shed angular momentum as they contract toward the main sequence, and will therefore begin to rotate more rapidly than their disked neighbors. Edwards et al. (1993) provided a key observational result in support of this paradigm: inferring the presence or absence of a circumstellar disk from a T Tauri star's dereddened $H-K$ color, Edwards et al. (1993) found that stars with substantial disks were uniformly slow rotators ( $P_{\text {rot }}>4$ days), while the period distribution for stars lacking disks spanned a broader range of periods and notably extended to significantly shorter periods (i.e., $1.5<P_{\text {rot }}<16$ ). Subsequent studies of rotation periods for optically visible $\mathrm{T}$ Tauri stars in nearby young clusters have reinforced this statistical connection between $\mathrm{T}$ Tauri disks and stellar rotation rates (e.g., Rebull et al. 2004; Cieza \& Baliber 2007; Irwin \& Bouvier 2009), with studies at infrared wavelengths providing a first glimpse of the rotation periods and spectroscopic $v$ sin $i$ measurements for embedded Class I protostars (e.g., Covey et al. 2005; MoralesCalderón et al. 2011).

Using Spitzer photometry to diagnose the presence or absence of a circumstellar disk around each TrES target, we have analyzed how the periods presented here can inform our understanding of the connection between pre-main-sequence rotation and circumstellar disks. We adopt a Spitzer [3.6]-[8] 1 colorcut to distinguish between objects which do have a disk (i.e., $[3.6]-[8]>1$ ) and those which do not (i.e., [3.6]-[8] $<1$ ). This classification is similar to those adopted in similar contexts by Rebull et al. (2006), Lada et al. (2006), and Le Blanc et al. (2011), and its ability to cleanly distinguish between stars with and without disks is evident in Figure 3, where few Taurus members lie close to this separation line. To enable a direct comparison with Figures 3 and 4 shown by Edwards et al. (1993), we plot in Figure 11 Spitzer [3.6] - [8.0] colors as a function of the logarithm of the TrES period for each of the T Tauri stars we classify as "Definitely Periodic" or "Possibly Periodic." While the sensitivity and accuracy of the observations used to infer each star's period and disk properties have improved significantly since the analysis of Edwards et al. (1993), the underlying result has not changed: stars with massive circumstellar disks are preferentially slow rotators ( $P_{\text {rot }}>4$ days), while diskless stars cover a broader range of periods, including slow rotators but also objects rotating much more rapidly (i.e., $P_{\text {rot }} \lesssim 1$ day).

The periods we measure here do not modify our qualitative understanding of the connection between stellar rotation and circumstellar disks, but they do provide a modest improvement in the statistical distinction between the rotation periods of disked and diskless $\mathrm{T}$ Tauri stars. We show in Figure 12 the rotation period distributions for Taurus members with and without disks, assuming periods from the prior literature (top panel) or using our new TrES period measurements (bottom panel), with the full distribution of Taurus rotation periods shown in each panel for completeness. Applying a K-S test to determine if the rotation periods of stars with and without disks 


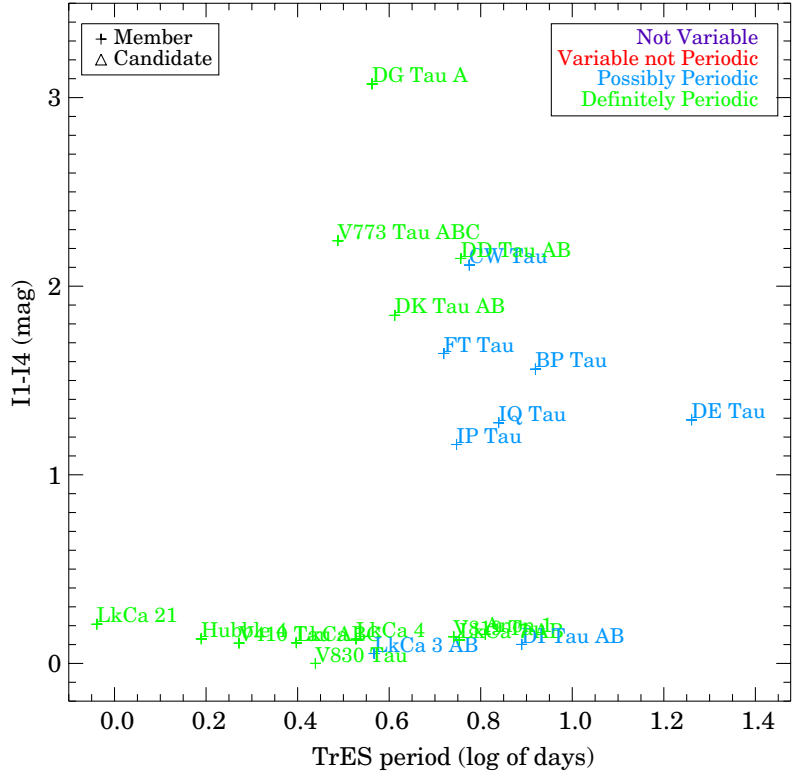

Figure 11. Following Figures 3 and 4 of Edwards et al. (1993), a plot of IRAC [3.6]-[8] color, a signature of primordial circumstellar disks, against the logarithm of the TrES rotation period for T Tauri stars that we classify as "Definitely Periodic" or "Possibly Periodic." While the sensitivity and accuracy of the observations used to infer each star's period and disk properties have improved significantly since the analysis of Edwards et al. (1993), the underlying result has not changed: stars with massive circumstellar disks are preferentially slow rotators $\left(P_{\text {rot }}>4\right.$ days), while diskless stars cover a broader range of periods, including slow rotators but also objects rotating much more rapidly (i.e., $P_{\text {rot }} \lesssim 1$ day).

(A color version of this figure is available in the online journal.)

could have been drawn from the same parent sample, we find that the new TrES periods provide a clearer difference between the two populations: the disk and diskless distributions have a $13 \%$ chance of being drawn from the same parent population if literature periods are used, but this drops to a $10 \%$ chance of a shared parent distribution when the most reliable sample of TrES periods is adopted.

The overall shapes of the disked and diskless rotation period distributions, however, do differ somewhat from what has been seen in other clusters such as NGC 2264 and the ONC. In those clusters, stars with circumstellar disks tend to rotate slowly, with a rotation period distribution peaking at $\sim 8$ days, while the diskless stars rotate more rapidly, peaking at $\sim 1-2$ days. Our Taurus sample, however, does not provide as clean a separation between these populations; we find that the period distribution of diskless stars contains peaks in both short $(P \sim 2-3$ days $)$ and long $(P \sim 7$ days) periods, while stars with disks populate a flatter unimodal population spanning $P \sim 3-7$ days. This may simply be a spurious result due to the relatively small number of stars in our sample, but it does motivate further monitoring of L1495 to confirm that these rotation periods are indeed correct, and the monitoring of additional areas within Taurus to further expand the sample of Taurus members with accurate rotation periods.

\section{SUMMARY}

We analyzed TrES light curves for YSOs in the L1495 dark cloud in Taurus using Spitzer photometry. The TrES/Spitzer catalog includes detections of 48 of 179 confirmed Taurus members in the TrES field. In addition, 26 objects identified by Rebull et al. (2010) as candidate Taurus memebers were also

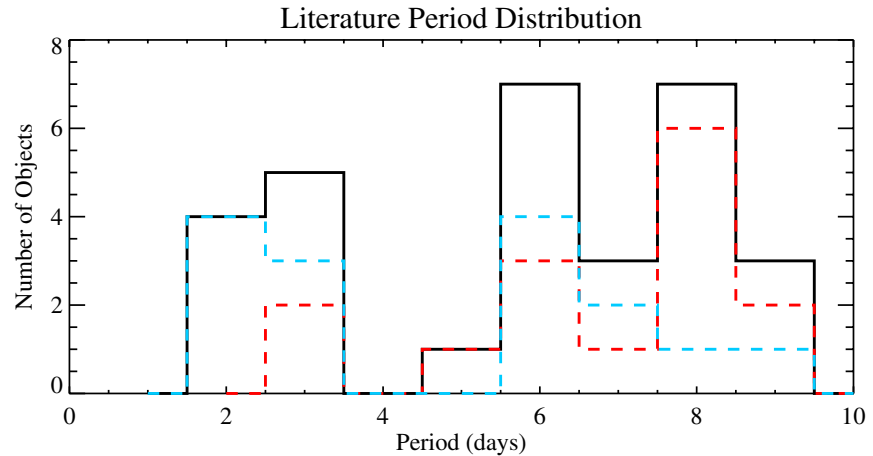

TrES Period Distribution

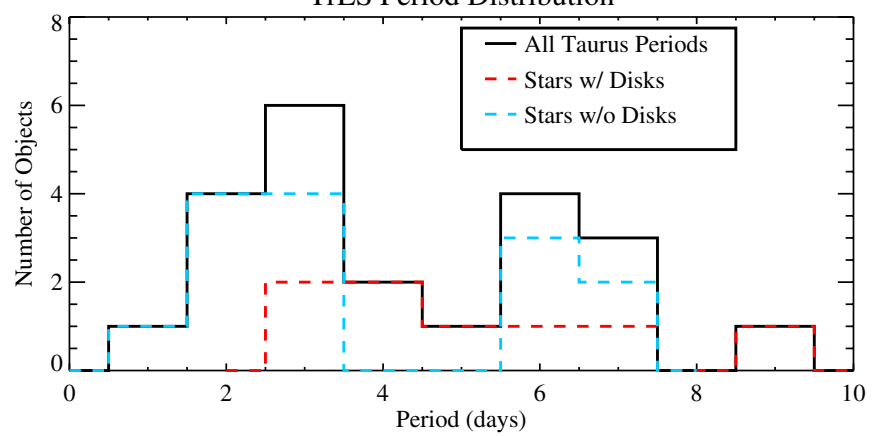

Figure 12. Period distributions of Taurus members with and without circumstellar disks, as determined from a Spitzer [3.6]-[8] = 1 color-cut. Top: period distributions assuming periods reported in the literature. Bottom: period distributions where TrES periods are adopted for all stars for which they have been measured, and where periods that are not recovered by TrES are eliminated. A K-S test confirms that the new TrES periods do not erase the distinction between the rotation period distributions of stars with and without circumstellar disks, with only a $10 \%$ chance that the two populations share the same parent distribution.

(A color version of this figure is available in the online journal.)

detected. Our analysis of the TrES light curves of these objects yielded the following results.

1. Consistent with previous photometric studies of pre-mainsequence stars, we find that Taurus members exhibit significantly more photometric variability than the surrounding field stars. We quantify each star's variability as the ratio of the standard deviation of the original light curve to the standard deviation of a light curve that has been smoothed by 9 or 1001 epochs. Known Taurus members typically demonstrate $\left(\sigma_{\text {orig. }} / \sigma_{9}\right)<2.0$, and $\left(\sigma_{\text {orig. }} / \sigma_{1001}\right)<5$, while field stars reveal $\left(\sigma_{\text {orig. }} / \sigma_{9}\right) \sim 3.0$ and $\left(\sigma_{\text {orig. }} / \sigma_{1001}\right) \sim 10$, as expected for light curves dominated by unstructured white noise.

2. We analyzed the TrES light curves for each detected Taurus YSO to identify periodic and aperiodic variability. Of the 74 known or candidate T Tauri stars with TrES light curves, we detect significant variability from 49 sources. We further classify these sources into 33 periodically variable sources (counting both the "Definitely Periodic" and "Possibly Periodic" categories) and 16 aperiodically variable sources. Using a quantitative metric to measure the amount of red and white noise in each light curve, and thereby assess the reliability of each period measurement, we identify 18 highly significant period measurements, and 15 periods in which we have lower confidence. Our measurements appear to be the first periods reported for 11 of the sources in our sample; of these, 8 are confirmed Taurus members. 
3. We find good agreement between the periods measured from the TrES light curves and those reported previously in the literature for the same objects. Periods have been previously reported for 14 of the 18 stars with the highest confidence TrES periods: the TrES period agrees with a prior measurement to within $1 \%$ for 11 of these sources; for two sources, the TrES light curves reveals that the prior period measurement was an alias of the true period. The agreement between the TrES period and that found in the literature is poorer for the 15 objects with lower confidence period measurements that we classify as "Possibly Periodic"; these lower confidence detections are biased toward longer periods, however, suggesting that longer period systems may exhibit greater levels of aperiodic variability as well, complicating the extraction of reliable period measurements.

4. As young stars typically exhibit elevated photometric variability, we identify 10 of the 26 candidate Taurus members as demonstrating variability levels consistent with being bona fide $\mathrm{T}$ Tauri stars.

5. K-S tests indicate that our TrES measurements bring Taurus' period distribution into better agreement with the period distributions measured within NGC 2264, NGC 6530, and the ONC. This increased agreement may largely reflect, however, the removal of long rotation periods that are detected at lower significance in Taurus, and which may be beyond the limits of detectability in more distant starforming regions.

6. Using Spitzer photometry to infer the presence or absence of circumstellar disks around each $\mathrm{T}$ Tauri star, we find that the TrES periods provide a clearer distinction between the period distributions of stars with and without disks, particularly considering the number of disk hosts with marginally detected, long $(5+$ days $)$ periods which are excluded from this comparison.

The authors thank Lynne Hillenbrand for helping motivate the TrES team to monitor L1495; Michael Meyer and Eric Mamajek for useful discussions that inspired this analysis of the data; Eric Feigelson, Adam Miller, and Joseph Richards for informative discussions of period detection techniques that would be robust against the presence of red noise; and Jerome Bouvier for noting that $v \sin i$ measurements may support $\mathrm{LkCa}$ 21 's sub-day period. We also thank the anonymous referee for thoughtful comments which spurred several improvements to the analysis and interpretation presented here.

K.R.C. acknowledges support for this work from the Hubble Fellowship Program, provided by NASA through Hubble Fellowship grant HST-HF-51253.01-A awarded by the STScI, which is operated by the AURA, Inc., for NASA, under contract NAS 5-26555. This material is based on work that was supported by NASA under grants NNG05GJ29G issued through the Origins of Solar Systems Program.

This research has made use of NASA's Astrophysics Data System Bibliographic Services, the SIMBAD database, operated at CDS, Strasbourg, France, the NASA/IPAC Extragalactic Database, operated by the Jet Propulsion Laboratory, California Institute of Technology, under contract with the National Aeronautics and Space Administration, and the VizieR database of astronomical catalogs (Ochsenbein et al. 2000). IRAF (Image Reduction and Analysis Facility) is distributed by the National Optical Astronomy Observatories, which are operated by the Association of Universities for Research in Astronomy, Inc., under cooperative agreement with the National Science Foundation.

The Two Micron All Sky Survey was a joint project of the University of Massachusetts and the Infrared Processing and Analysis Center (California Institute of Technology). The University of Massachusetts was responsible for the overall management of the project, the observing facilities, and the data acquisition. The Infrared Processing and Analysis Center was responsible for data processing, data distribution, and data archiving.

\section{REFERENCES}

Agüeros, M., Covey, K., Lemonias, J., et al. 2011, ApJ, 740, 110

Alard, C. 2000, A\&AS, 144, 363

Alonso, R., Brown, T. M., Charbonneau, D., et al. 2007, in ASP Conf. Ser. 366, Transiting Extrapolar Planets Workshop, ed. C. Afonso, D. Weldrake, \& T. Henning (San Francisco, CA: ASP), 13

Alonso, R., Brown, T. M., Torres, G., et al. 2004a, ApJ, 613, L153

Alonso, R., Deeg, H. J., Brown, T. M., \& Belmonte, J. A. 2004b, Astron. Nachr., 325,594

Audard, M., Briggs, K. R., Grosso, N., et al. 2007, A\&A, 468, 379

Bouvier, J., Cabrit, S., Fernandez, M., Martin, E. L., \& Matthews, J. M. 1993, A\&A, 272, 176

Bouvier, J., Covino, E., Kovo, O., et al. 1995, A\&A, 299, 89

Bouvier, J., Wichmann, R., Grankin, K., et al. 1997, A\&A, 318, 495

Carter, J. A., \& Winn, J. N. 2009, ApJ, 704, 51

Cieza, L., \& Baliber, N. 2007, ApJ, 671, 605

Collier Cameron, A., Davidson, V. A., Hebb, L., et al. 2009, MNRAS, 400, 451

Covey, K. R., Greene, T. P., Doppmann, G. W., \& Lada, C. J. 2005, AJ, 129,2765

Covey, K. R., Hillenbrand, L. A., Miller, A. A., et al. 2011, AJ, 141, 40

Cranmer, S. R. 2008, ApJ, 689, 316

Cumming, A., Marcy, G. W., \& Butler, R. P. 1999, ApJ, 526, 890

Delorme, P., Collier Cameron, A., Hebb, L., et al. 2011, MNRAS, 413, 2218

Devor, J., Charbonneau, D., O’Donovan, F. T., Mandushev, G., \& Torres, G. 2008a, AJ, 135, 850

Devor, J., Charbonneau, D., Torres, G., et al. 2008b, ApJ, 687, 1253

Dunham, E. W., Mandushev, G. I., Taylor, B. W., \& Oetiker, B. 2004, PASP, 116,1072

Edwards, S., Strom, S. E., Hartigan, P., et al. 1993, AJ, 106, 372

Fazio, G. G., Hora, J. L., Allen, L. E., et al. 2004, ApJS, 154, 10

Finkbeiner, D. P., Padmanabhan, N., Schlegel, D. J., et al. 2004, AJ, 128, 2577

Frescura, F. A. M., Engelbrecht, C. A., \& Frank, B. S. 2008, MNRAS, 388,1693

Grankin, K. N. 1992, Inf. Bull. Var. Stars, 3720, 1

Grankin, K. N. 1993, Inf. Bull. Var. Stars, 3823, 1

Grankin, K. N. 1997, in IAU Symp. 182, Herbig-Haro Flows and the Birth of Stars, ed. F. Malbet \& A. Castets (Cambridge: Cambridge Univ. Press), 281P

Grankin, K. N., Bouvier, J., Herbst, W., \& Melnikov, S. Y. 2008, A\&A, 479, 827

Güdel, M., Padgett, D. L., \& Dougados, C. 2007, in Protostars and Planets V, ed. B. Reipurth, D. Jewitt, \& K. Keil (Tucson, AZ: Univ. Arizona Press), 329

Guieu, S., Dougados, C., Monin, J., Magnier, E., \& Martín, E. L. 2006, A\&A, 446,485

Hamilton, C. M., Herbst, W., Shih, C., \& Ferro, A. J. 2001, ApJ, 554, L201

Hartman, J. D., Gaudi, B. S., Holman, M. J., et al. 2008, ApJ, 675, 1254

Hartmann, L. 2002, ApJ, 566, L29

Hartmann, L., Hewett, R., Stahler, S., \& Mathieu, R. D. 1986, ApJ, 309, 275

Hartmann, L., Kenyon, S., \& Hartigan, P. 1993, in Protostars and Planets III, ed. E. H. Levy \& J. I. Lunine (Tucson, AZ: Univ. Arizona Press), 497

Henderson, C. B., \& Stassun, K. G. 2012, ApJ, 747, 51

Hillenbrand, L. A., Miller, A. A., Covey, K. A., et al. 2012, AJ, submitted (arXiv:1208.2066)

Horne, J. H., \& Baliunas, S. L. 1986, ApJ, 302, 757

Irwin, J., Berta, Z. K., Burke, C. J., et al. 2011, ApJ, 727, 56

Irwin, J., \& Bouvier, J. 2009, in IAU Symp. 258, ed. E. E. Mamajek, D. R. Soderblom, \& R. F. G. Wyse (Cambridge: Cambridge Univ. Press), 363

Joy, A. H. 1945, ApJ, 102, 168

Kenyon, S. J., Gómez, M., \& Whitney, B. A. 2008, in Handbook of Star Forming Regions, Volume I, ed. B. Reipurth (San Fransisco, CA: Astronomical Society of the Pacific), 405

Knott, G. 1891, Observatory, 14, 97 
Koen, C. 1990, ApJ, 348, 700

Kundurthy, P., Meyer, M. R., Robberto, M., Beckwith, S. V. W., \& Herbst, T. 2006, AJ, 132, 2469

Lada, C. J. 1987, in IAU Symp. 115, Star Forming Regions, ed. M. Peimbert \& J. Jugaku (Cambridge: Cambridge Univ. Press), 1

Lada, C. J., Muench, A. A., Luhman, K. L., et al. 2006, AJ, 131, 1574

Le Blanc, T. S., Covey, K. R., \& Stassun, K. G. 2011, AJ, 142, 55

Lomb, N. R. 1976, Ap\&SS, 39, 447

Lynds, B. T. 1962, ApJS, 7, 1

Makovoz, D., \& Marleau, F. R. 2005, PASP, 117, 1113

Mandushev, G., O’Donovan, F. T., Charbonneau, D., et al. 2007, ApJ, 667, L195

Mandushev, G., Torres, G., Latham, D. W., et al. 2005, ApJ, 621, 1061

Matt, S., \& Pudritz, R. E. 2005, ApJ, 632, L135

Miller, A. A., Hillenbrand, L. A., Covey, K. R., et al. 2011, ApJ, 730, 80

Mohanty, S., \& Shu, F. H. 2008, ApJ, 687, 1323

Monin, J.-L., Guieu, S., Pinte, C., et al. 2010, A\&A, 515, A91

Morales-Calderón, M., Stauffer, J. R., Hillenbrand, L. A., et al. 2011, ApJ, 733,50

Nguyen, D. C., Jayawardhana, R., van Kerkwijk, M. H., et al. 2009, ApJ, 695,1648

Norton, A. J., Wheatley, P. J., West, R. G., et al. 2007, A\&A, 467, 785

Ochsenbein, F., Bauer, P., \& Marcout, J. 2000, A\&AS, 143, 23

O’Donovan, F. T., Charbonneau, D., Bakos, G. Á., et al. 2007, ApJ, 663, L37

O’Donovan, F. T., Charbonneau, D., \& Kotredes, L. 2004, in AIP Conf. Ser 713, The Search for Other Worlds, ed. S. S. Holt \& D. Deming (Melville, NY: AIP), 169

O’Donovan, F. T., Charbonneau, D., Mandushev, G., et al. 2006, ApJ, 651, L61

Osterloh, M., Thommes, E., \& Kania, U. 1996, A\&AS, 120, 267

Padgett, D. L., Rebull, L. M., McCabe, C.-E., et al. 2008, Taurus Legacy Survey Document (P08) http://irsa.ipac.caltech.edu/data/SPITZER/Taurus/docs/ delivery_doc2.pdf
Padmanabhan, N., Schlegel, D. J., Finkbeiner, D. P., et al. 2008, ApJ, 674, 1217

Percy, J. R., Grynko, S., Seneviratne, R., \& Herbst, W. 2010, PASP, 122, 753

Pont, F., Zucker, S., \& Queloz, D. 2006, MNRAS, 373, 231

Rebull, L. M., Hillenbrand, L. A., Strom, S. E., et al. 2000, AJ, 119, 3026

Rebull, L. M., Padgett, D. L., McCabe, C., et al. 2010, ApJS, 186, 259

Rebull, L. M., Stauffer, J. R., Megeath, S. T., Hora, J. L., \& Hartmann, L. 2006, ApJ, 646, 297

Rebull, L. M., Wolff, S. C., \& Strom, S. E. 2004, AJ, 127, 1029

Reegen, P. 2007, A\&A, 467, 1353

Rieke, G. H., Young, E. T., Engelbracht, C. W., et al. 2004, ApJS, 154, 25

Rydgren, A. E., \& Vrba, F. J. 1983, ApJ, 267, 191

Rydgren, A. E., Zak, D. S., Vrba, F. J., Chugainov, P. F., \& Zajtseva, G. V. 1984, AJ, 89, 1015

Scargle, J. D. 1982, ApJ, 263, 835

Scholz, A. 2012, MNRAS, 420, 1495

Shang, H., Allen, A., Li, Z.-Y., et al. 2006, ApJ, 649, 845

Shu, F., Najita, J., Ostriker, E., et al. 1994, ApJ, 429, 781

Skrutskie, M. F., Cutri, R. M., Stiening, R., et al. 2006, AJ, 131, 1163

Southworth, J. 2008, MNRAS, 386, 1644

Stelzer, B., Fernández, M., Costa, V. M., et al. 2003, A\&A, 411, 517

Stetson, P. B. 1987, PASP, 99, 191

Stetson, P. B. 1992, in ASP Conf. Ser. 25, Astronomical Data Analysis Software and Systems I, ed. D. M. Worrall, C. Biemesderfer, \& J. Barnes (San Francisco, CA: ASP), 297

Sturrock, P. A., \& Scargle, J. D. 2010, ApJ, 718, 527

Vaughan, S. 2010, MNRAS, 402, 307

Vaughan, S., Bailey, R. J., \& Smith, D. G. 2011, Paleoceanography, 26, PA4211

Vio, R., Andreani, P., \& Biggs, A. 2010, A\&A, 519, A85

Vogel, S. N., \& Kuhi, L. V. 1981, ApJ, 245, 960

Vrba, F. J., Herbst, W., \& Booth, J. F. 1988, AJ, 96, 1032

Werner, M. W., Roellig, T. L., Low, F. J., et al. 2004, ApJS, 154, 1 